\title{
Estudios de Metabolismo Básico en el Perú
}

POR EC

Doctor Alberto Hurtado i.

(Introducción)

En plena era funcional, cuando los conccimientos patológicos y bacteriológicos que tantísimo han contribuído al progreso de la Medicina aceptan hoy como rival el concepto funcional como explicación de fenómenos fisiológicos y clínicos, no es de extrañarse que en las dos últimas décadas el campo de la $\mathrm{Me}$ tabolimetría se hava enriquecido con innumerables contribuciones que han establecido firmemente el valor de estos estudios.

El oscuro campo de la Endocrinología en su faz más interesante, tal como es la actividad de la glándula tiroide, recibe un enorme impulso cuando no solamente es posible medir la actividad de esta glándula, sino también fundar un pronóstico y guiar el tratamiento, sea éste médico o quirúrgico. La dietéti- . ca abandona su estado de empirismo, y, ya sea en estados normales o patológicos, permite su establecimiento en términos precisos de calorías, con arreglo a los diversos alimentos y es posible predecir los fenómenos de asimilación, y quizás su adelanto más grande lo encontramos en la dietética infantil, siendo posible también conocer los requerimientos calóricos del organismo que crece, $y$ establecer la alimentación artificial sobre bases precisas y científicas. Entidades clínicas tales como la diabetes y la obesidad, aclaran mucho de lo misterioso de los fenómenos intermediarios metabólicos, y estos nuevos conocimientos establecen sobre bases más racionales la terapéutica. La admirable regulación de la temperatura en el organismo y la 
alteración de este mecanismo durante los estados febriles reciben una explicación adecuada; y si seguimos más adelante estudiando el efecto de ciertas drogas sobre el organismo, y de otros tantos estados moriosos, nos convencemos del inmenso impulso que el estutio de Metabolimetria ha introducido en la clínica, y de lo fascinante de estos estudios que abarcan la investigación de la actividad total del organismo en su cualidad más fundamental de ser viviente, tal como es la producción de calor y energia que requiere el consumo de oxígeno y la eliminación de anhidrido carbónico.

Pero no solamente es en el campo de la clínica donde encontramos el interés de estos estudios. En los últimos años cierta faz de la Medicina Preventiva ha adquirido una enorme importancia, y vemos cómo las investigaciones actuales se orientan hacia el estudio de los caracteres constitucionales de los individuos, de sus cualidades de resistencia e inmunidad, y trata de establecer las defensas naturales del organismo, teniendo como lema la prevención antes de que la Patología, en sus diversos aspectos, adquiera el rol preponderante. $Y$ entre estos estudios se destaca como punto de enorme interés e importancia el que investiga los caracteres raciales de un pueblo, el que establece sus rasgos antropométrices creando un tipo aparte físicamente, $y$ que profundizando aún más establece sus condiciones diferentes de inmunidad, de resistencia y debilidad a ciertas afecciones, estudiando el porqué de estas diferencias y la posible aplicación de sus cualidades favorables a otras razas. $Y$ entre nosotros existe atrayente y virgen el estudio de la raza indígena, de esta raza que la fantasía popular imagina decadente, física y moralmente, producto espontáneo de nuestras montañas y del. campo, alejada de la medicina actual, que vive en sus alturas laborando pacientemente y que al bajar a nuestras ciudades, cambiando totalmente sus hábitos de vida, nos da aquel triste enfermo de nuestros Hospitales, anemizado y palúdico o caído en las garras de la gran plaga blanca. $\mathrm{Y}$ sin embargo, cuando llegamos a sus moradas, en sus tierras nativas, nos sorprendemos ante ciertos caracteres únicos que ditinguen a esta admirable raza. De estatura menos que mediana, de peso algo elevado para su italla, de un enorme tórax con caracteres de enfisematoso, venos a estos individios trabajar sin descanso por largas horas, con alimentación insuficiente en muchos casos, pero siem-. 
pre poseyendo una resistencia física que sorprende, resistencia que se hace aún más admirable cuando los contemplamos en su vida ordinaria en las alturas, a muchos miles de pies sobre el nivel del mar, donde las necesidades fisiológicas sufren por la falta de oxigenación necesaria, y sin embargo no parecen sufrir de las fatigas y sintomas que el organismo exhibe al ascender a estos lugares. Soldado admirable por su resistencia y cualidades de adaptación, es indudable que el estudio metabólico de esta raza indigena debe poseer una atracción especial, pues es lógico suponer que este individuo físicamente diferente quizás posea un grado de actividad orgánica distinta, y que viva en un plano menor de actividad celular que signifique una mayor economía en el consumo de oxígeno con igual o mayor producción de energía.

$Y$ este estudio posee no sólo los caracteres atractivos de un campo virgen, sino también la importancia científica del establecimiento de valores normales de metabolimetría en esta raza, pues en la actualidad sólo poseemos los aplicados a las razas europeas y americanas, y ya que hemos visto la importancia de dichos estudios en la clínica, es indispensable conocer ante todo los límites normales de variación de metabolismo básico en estos individuos antes de estudiar las variaciones patológicas, e igualmente estudiando esie aspecto normal inquirir sobre las necesidades calóricas de esta raza, que arrancada de sus hogares es sometido a regímenes dietéticos diferentes; $y$, si proseguimos más adelante, podemos considerar cuán impontante es el establecimiento de estos vaiores normales al contemplar tantas poblaciones que sufren de las garras del bocio endémico, afección que como bien sabemos puede transformarse en un bocio patológico cuya actividad debe medirse en términos calóricos antes de proceder a su tratamiento.

Este estudio posee singular atracción e importancia no sólo clesde el punto de vista racial, y como uno de los caracteres individuales del indio en nuestro pais, donde existen poblaciones al nivel del mar y en las altas cadenas de los Andes; donce el indio vive en zonas templadas, frías y calurosas; donde es posible estudiar la influencia del clima y costumbres en el metabolismo básico de un individuo, sino que se hace tambiën importante e imperativo, ya que quizás los valores que establecemos al nivel del mar son inaplicables en nuestras montañas, $\odot$ 
cuando nos acercamos a las regiones candentes del Norte sus habitantes viven en otro nivel metabólico de adaptación, como lo afirman algunos investigadores.

$Y$ este trabajo nacionalista, novedoso y atrayente, to hemos abordado con entusiasmo y fé. Hemos llegado hasta los mismos indígenas que viven en saquellas alturas de muestros Andes, estudiando su metabolismo básico, sus necesidades caloricas, sus hábitos de vida; hemos comparado su actividad orgánica con la de habitantes de la costa llevados a esas alturas; hemos estudiado al indio traído al nivel del mar cuando abandona sus costumbres primitivas, tipo que ingresa a la sala de nuestros Hopitales, y, finalmente, hemos estudiado el metabolismo en el trópico, donde la actividades físicas ordinarias sufren la laxitud de un clima candente y los nativos caen en las garras de una vida lánguida $\mathrm{y}$ monótona.

En las páginas que siguen damos el resultado de estos es-tudios y la posible aplicación de los valores normales que se usan en otros centros. $E_{S}$ indudable que este trabajo representa un estudio inicial en el fecundo campo de la Metabolimetría, y tenemos la esperanza de que sirva de punto de partida a otras investigaciones más amplias e importantes. Es para nosotros una gran satisfacción expresar nuestro agradecimiento a todos los que nos han prestado ayuda material y espiritual. Sea nuestro primer recuerdo a ba memoria del Profesor Francis W. PEABody, cuya temprana desaparición es una pérdida irreparable y para quien nuestra gratitud es inmensa, ya que recordamos con emoción sus cariñosas enseñanzas personales y el interés que supo inculcarnos por estos estudios. Al Profesor Carlos MONGE, por su constante aliento y facilidades prestadas; al Dr. Gerardo Alarco, por poner a nuestra disposición los Servicios de Sanidad Militar en los diversos lugares visitados; al Dr. Enrique Rondós, por practicar las determinaciones en la Oroya, y, finalmente, a todos los, que nos favorecieron con su consejo y ayruda. incluyendo a aquellos que se prestaron a la determinación de metabolismo, a todos y cada uno de los cuales expresamos nuestro más sincero agradecimiento. 


\section{Breves nociones sobre Metabolimetria}

Al investigar la historia de las diferentes namas en Medicina, siempre encontramos la figura descollante de un investigador que echa las bases de los futuros conocimientos. En Metabolimetría tal honor pertenece a LAvoISIER, genio francés, quien en I\$49 describió minuciosamente la producción de oalor y energía en el organismo, explicó $I_{a}$ combustión de oxígeno y la eliminación de anhidrido carbónico, y aun adelantándose muchos años a los conocimientos actuales, construyó un aparato con el cual pudo medir las actividades metabólicas del hombre y sus variaciones bajo la influencia de la ingestión de alimentos, de la temperatura y del ejercicio.

Sobre los conocimientos enunciados por Lavorsier se han desarrollado todos los adelantos de esta ciencia, y a sus claros principios poco o nada ha tenido que agregarse. Pocos años antes, dos hechos importantes se distinguen: el descubrimiento del equivalente mecánico, por Joute, en 1842 , y la enunciación de la ley de conservación de la energía, en 1845, por MAYer y HelmHOLTz. En la época de LavoISIER, otros dos investigadores franceses, Regnault y Rieset, publicaron la descripción de un aparato ,con el que les fué posible medir el metabolismo de los animales calculando el oxígeno consumido y el anhídrido carbónico eliminado, e igualmente trataron de establecer idénticos aparatos en los Hospitales de París para estudiar làs actividades metabólicas en las diferentes afecciones clínicas, evidenciando así el temprano interés por aplicar estos conocimientos en la $\mathrm{Me}$ dicina.

Años más tarde, el gran químico alemán LIEBIG sumó a los conocimientos existentes estudios detenidos de los cambios químicos y asimilativos que sufren los alimentos al ser incorporados al organismo, y los dividió en las tres grandes categorías de proteínas, grasas y carbohidratos, Pettenkoffer y Voit, en 1867 , construyeron un calorímetro y practicaron los primeros estudios completos de Metabolimetría en clínica, y la literatura dejada por estos dos investigadores constituve en realidad la primera fuente de consulta que tenemos a nuestra disposición. ZUNTZ y GEPPERT aportaron una brillante contribución al fabricar un aparato con el cual era posible calcular las actividades 
metobólicas nediante la observación en contos periodos del intercambio de gases (oxjgeno y anhidrido carbónico), y fué MAgrivus LeVy, años más tarde, quien, mediante el uso de este aparato, impulisó enormemente el estudio de los desórdenes metabólicos en diversas afecciones.

No obstante todas estas magníficas investigaciones y estudios, sólo en la presente centuria vemos realizada la vieja idea de lia importancia de la Metabolimetría en clínica; faltaba a los previos investigadores lo más esencial, tal como es el establecimiento de los valores normales calóricos, base indispensable para comparar las alteraciones en Patología. Las previas investigaciones se referían a medidas hoy no usadas, y el estudio comparativo de las alteraciones metabólicis aun no se había realizado. Fué, pues, hace muy pocos años que diversos investigadores, principalmente en Estados Umidos, sentaron las bases de la Metabolimetría al establecer las cirras calóricas que corresponder a un individuo normal, y fundándose en estos descubrimientos, la literatura mé́lica en los últimos años se encuentra llena de contribuciones importantes, pudiéndose en la actualidad. afirmar el inmenso valnr de estos estudios y su definitiva incorporación en lay clínica. Means, Aub, Du Bors, Benedict, HaRRIS $y$ otros muchos laan hecho importantes estudios en este: sentido.

Por metabolismo en general se entiende todos los cambias. químicos que sufren los productos de la digestión, después de ser abscrbidos del canal intestinal. Algunos de estos productos son almacenados como tales o después de sufrir ciertas transformaciones; otros sufren un desdoblamiento en productos más. simples y eliminados del organismo, mientras que, fimalmente, los más importantes son transformados en el protoplasma viviente de los tejidos.

Todos estos cambios químicos pueden ser agrupados en las dos grandes divisiones del metabolismo, y podemos considerar los fenómenos catabólicos, o sea la reducción de productos complejos a otros más simples, y los fenómenos de anabolismo, - sea la construcción de substancias complejas de productos más. 
simples. Estudiando estas transformaciones metabólicas se puede seguir cada substancia individualmente, desde su ingreso al organismo hasta que sea convertida en producto final de almacenamiento o eliminación; sin embargo, no. es este el método que nos interesa, pues nuestro fin es el estudio total del metabolismo, y debe nos considerar al cuerpo humano como un enorme aparato de combustión en el que las diversas substancias alimenticias son oxidadas, hidrolizadas y sometidas a la acción de diversos fermentus con producción de energía potencial, que se transforma en parte en trabajo mecánico, pero en su gran mayoría en calor que puede ser medido y expresado en términos de calorías.

La producción de este calor es de urgente necesidad para el organismo, que a temperatura, ordinaria pierde calor rdpidamente, y condición esencial para la vida de un organismo es el mantenimiento de una temperatura apropiada; más o menos un cuarto del calor total del organismo se pierde por evaporación de agua de la piel y pulmones, e igualmente hay pérdida en el transporte de calor de los órganos interiores a la piel por medio de la sangre.

Considerando el metabolismo en general, es interesante calcular cuánto del calor producido se deriva de la actividad de los órganos internos. KROGH cree que el metabolismo de los riñones es responsable del $5 \%$ del metabolismo total, que la acción cardíaca contribuye en un $4 \%$ y los movimientos respiratorios alrededor de Io\%, considerando que de la actividad total de todos los órganos depende un $25 \%$ del metabolismo del orgunismo. Esto nos deja la conclusión de que tres cuartas partes del calor producido proviene de la oxiclación de los tejidos en descanso, y es muy probable gue el toino nniscular sea en gran parte responsable del metabolismo básico de un individuo.

Hemos dicho que nuestro interés radica en la medida del metabolismo total del organismo expresado en términios de calor. Para llevar a cabo esta medida tenemos dọ grandes métodos a nuestra disposición. En primer lugar podemos medir directamente el calor eliminarlo del cuerpo por medio de la radiación, conducción, convección y evaporización del agua de la piel y pulmones. Este es el método de calorimetría directa. La producción de calor es la suma del calor eliminado y la corrección por el cambio de temperatura del cuerpo durante el período de observación. Este calor producido es medido en aparatos especiales llamados calorímetros, en los que el oalor es absorbido por me- 
dio de una coritiente de agua que circula en tubería especiai, calculando la cantidad y temperatura de esta agua antes y después de entrar al calorímetro, y a esto se agrega el calor disipado en la evaporización del agua. Para el cálculo de la cantidad de agua evaporizada es necesario analizar la cantidad de agua en el aire, y esto se efectúa haciendo circular el aire a través de una botella con gránulos de cloruro de calcio o ácido sulfúrico, o determinando directamente el grado de humedad por medio de termómetros.

El segundo método que tenemos a nuestra disposición es el de calorimetria indirecta, o sea el cálculo del metabolismo total del organismo por medio de la determinación del oxígeno consumido y del anhídrido carbónico eliminado en un tiempo dado, ya que sabemos cue el intercambio de estos grases es el fenómeno esencial de la actividad celular y puede constituir un indice de esta activided. Para determinar de una manera precisa el metabolismo por medio de este método es necesario calcular la clase y cantidad de substancia alimenticia que se quema en el organismo, y esto se verifica determinando las productos finales de estas substancias. El cálculo de ritrógeno en la orina nos da una idea del metabolismo de las proteínas, y la determinación del oxígeno consumido y el anhídrido carbónico eliminado por los pulmones da un índice del metabolismo de los carbohidratos y grasas, constituyendo la relación entre los dos el coeficiente respiratorio que varía según la substancia quema da durante el período de observación; este coeficiente respiratorio igualmente es influenciado por la conversión de una substanica alimenticia en otra en el organismo.

Para medir casi exactamente la cantidad de calor producida en el organismo por medio de este último método, es indispensable la medida de los dos gases (oxígeno y anhídrido carbónico), ya que la cantidad de oxígeno consumido o anhídride carbónico eliminado varía según la clase de alimento que se oxida y tenemos que el valor calórico de un litro de oxígeno es de 4.686 cuando las materias grasas son quemadas, mientras que cuando los carbohidratos son oxidados el valor calórico del litro de oxígeno es de 5.047 calorias.

Los resultados del cálculo del metabolismo total del organismo por medio de estos dos métodos (calorimetría directa e indireota) son casi iguales, con variaciones menores de uno por 
ciento, y. por consiguiente es indiferente el método adoptado para la determinación del metabolismo.

Solsre los principios ya enunciados se han construido innumerables tipos de aparatos cuya descripción sería larga e inútil. En general se pueden dividir en dos grandes grupos, o sea las cámaras respiratorias donde el individuo cuyo metabolismo se desea medir es colocado, y el calor medido en observaciones que cubren largos períodos; y los numerosos aparatos más pequeños a los curales el individuo metabolizado es conectado por medio de mangueras o máscaras apropiadas. Casi todos estos aparatos usan el método de calorimetría indirecta, y las muestras de aire son analizadlas en los aparatos de HALDANE, HenDERSON o CARPENTER para el cáleulo de los gases respiratorios. Entre estos aparatos podemos citar los de Peten kofferVort, Rubner, Atwater-Rosa, Benedict, ZuntZ_Geppert, Tissor, Dovglas y otros muchos.

Sin embargo, últimamente se ha introducido en la clínica el uso de aparatos más sencillos y de más fácil manejo, en los cuale; el metabolismo es estudiado mediante el simple cálculo de runo de los gases ya mencionados. En estos aparatos es más ventajosa y precisa la medición del oxígeno consumido, pues la eliminación de anhidrido carbónico sufre grandes variaciones con anormalidades de la respiración, y si hay un exceso de ventilación pulmonar durante la prueba metabólica resulta una mayor eliminación de anhídrido carbónico que la que realmente proviene de la oxidación de los tejidos. El cálculo, pues, del oxígeno consumido durante un período dado nos da una mejor idea del metabolismo del organismo, y tenemos a nuestra disposición muchos aparatos en los cuales la determinación de este gas constituye la medida metabólica, que se lleva a cabo usando el método gráfico de registro de respiraciones, como veremos más adelante. De los aparatos más conocidos que se usan en diferentes clínicas, podemos citar los de Sanborn, Benedict-Roth, Benedict, KROGH, JoNES, etc. El uso de estos aparatos, aunque extremadamente sencillo, requiere un fuerte training, y en manos de técnicos competentes los resultados de estas pruebas por medio de los aparatos citados en último lugar, pueden alcanzar una variación de sólo $2 \%$ de los métodos más precisos. Sin embargo, debemos insistir en la preparación especial de los que usan estos aparatos: el escape de gases es frecuente y la cooperación total del enfermo es 
esencial, siéndolo también, sobre todo, la honestidad de desechar enteramente aquellos resultados sobre los que existe la menor duda, ya que hay un estrecho margen entre to normal y lo patológico.

"El gran peligro proviene de los resultados de los que poseen en training deficiente, $u$ observadores sin cridado que no toman las debidas precauciones para asegurar la exactitud de sus determinaciones. Basar un diagnóstico sobre los resultados de una de estas pruebas de metabolismo, en la que innumerables fuentes de error pasan desapercibidas, es un abuso a la ciencia, un insulto al arte clínico y un crimen contra el enfermo". (Mc. CANN).

La producción de calor en el organismo, el consumo de oxígeno y eliminación de anhídrido carbónico, no solamente están sujetos a variaciones segín ciertos factores individuales como veremos más adelante, sino que igualmente son influenciados por factores extraños, tales como la ingestión de los alimentos $y$ el ejercicio muscular. Investigaciones han demostrado que cualquier actividad física, por pequeña que sea, produce un aumento de metabolismo que está en proporción con el grado de actividad desarrollabr; aun la diferencia de estar sentado o echado en cama produce un cambio en la producción calórica, y es evidente entonces que los requerimientos calóricos de un individuo varían inmensamente según la ocupación a que esté dedicado, ya que el gasto de energía de un individuo de vida sedentaria es mucho menor que el de otro entregado a una vida activa.

La' ingestión de alimentos igualmente influencia el metabolismo, y después de ésta se ha demostrado un aumento que dura varias horas. La causa de este aumento es materia de discusión, y RUBNER cree que depende de la serie de transformaciones químicas que los mismos alimentos sufren al ser incorporados al organismo, mientras que LUsk mantiene la idea de que se debe principalmente a una mayor actividad metabólica de las mismas células de los tejidos, haciendo resaltar la acción dinámica específioa. que consiste en un aumento de actividad celular causada por un estímulo específico de las moléculas alimenticias, siendo este aumento más marcado con los alimentos proteicos. 
Si el metabolismo de un individuo varía con la ingestión de alimentos y con cualquier actividad física, es, pues, indudable que para establecer un estudio comparativo entre individuos normales o patolóyicos, es preciso eliminat estas dos fuentes de variaciones. Por consiguiente, en la clínica el metabolismo de un individuo es determinado en completo estado de reposo y por lo menos catorce horas después de la última comida. El metabolismo así determinado se denomina Metabolismo Básico. Todos nuestros estudios se refieren a la producción calórica así determinada, y toda referencia en la literatura igualmente se refiere al metabolismo en iguales condiciones. Sin embargo, la producción calórica en completo estado de reposo y en ayumas no es la menor, pues durante el sueño y en las primeras horas de la mana na cualquier individuo se encuentra en su nivel más bajo de metabolismo.

No obstante, al comparar la producción calórica de diversos individuos, vemos que también existen variaciones que dependen de ciertos factores subjetivos que discutiremos más adelante.

La edad influencia enormemente la actividad metabólica del organismo; interesantes estudios en los fetos de animales han demostrado que el metabolismo por unidad de peso es bastante alto en los embrios, siendo mayor al tiempo de la diferenciación. En los instantes prematuros, cuya vida es asaz difícil y precaria de sostener, el metabolismo es bastante bajo. En el infante normal, recién nacido, el metabolismo es más bajo que en la edad adulta si establecemos el área de superficie como término de comparación; sin embargo, aumenta rápidamente, alcanzando su mayor valor entre el primero y segundo año, cuando la producción calórica proporcionalmente alcanza su nivel más alto. A partir de esta edad desciende paulatinamente, llegando a su nivel más bajo en edad avanzada. Las determinaciones de metabolismo durante los primeros años de vida constituyen uno de los capítulos más intereantes de la Metabolimetría, ya que podemos considerar las dificultades de determinar el nivel metabólico a esa edad. Algunos investigadores creen que inmediatamente antes de la pubertad, en hambres $y$ mujeres, hay un pequeño aumento de metabolismo, y que este se debe al completo desarrollo del organismo, tanto físicamente como en sus diversos aspectos endocrinológicos. El aumento de metabolismo en 
los pequiños, posiblemente está asociado al estimulo específico del crecimiento, y KRogh cree que casi se deje exclusivamente al desarrollo del sistema muscular.

El sexo igualmente influencia el metabolismo, aunque en menor grado. Desde las primeras investigaciones se pudo demostrar que la producción calórica es menor alrededor de 5\% en las mujeres, y que esta menor producción calórica también se puede demostrar en los primeros años de vida. Indudablemente que ni las glándulas sexuales, ni la difereincia en desarrllo muscular explican este cambio de metabolismo en los dos sexos, pues estos dos factores no están presentes en la infancia, cuando el desarrollo muscular es el mismo y las glándulas sexuales no han evidenciado su actividad.

Es interesante consignar aquí la constancia de! metabolismo en un misno individuo; diversa; investigaciones han demestrado cue la producción calórica so nantiene igual día a día, fenómeno anćlogo al manteniniento del pulso. respiraciones, tensión arterial, etc., y que constituye un dato importante en el estudio comparativo del metabolismo.

Al iniciarse los modernos estudios de Metaljolimetría, tratrando de estabiecer los valores normales de producción calórica, pudo demostrarse que ésta no solamente varía con la edad y sexo, sino que era preciso referirla a alguna medida física individual, pues el metabolismo de diferentes individuos, aunque nornales y de la misma edad y sexn, era completanente diferente. Los primeros investigadores referían sus determinacines al peso de cada individuo, pero bien pronto se evidenció la inexactitud de este procedimiento, $y$ es por estc motivo quie las primeras referencias que tenemos en la literatura acerca de determinaciones en diferentes afecciones cinicas carecen hoy da valor.

SarRus y Rameaux, en 1838 , adelantaron la idea de que el metabolismo básico de un individuo es proporcional a su área de superficie, y ya que la temperatura del cuerpo se mantiene constante, debe haber una relación entre la producción y eliminación como evidentemente existe. En IS\&3, RuBNer y RICHET demostraron lo exacto de esta afirmación. y desde entonces innumerables trabajos han probado que el metalsolismo de un inclividuo es proporcional a su área de supe:ficie. Por muchos años la fórmula de MEefr sirvió para calcular el área cle super- 
ficie, que se obtenía elevando el peso (en lojlogramos) a la potencia 2/3. Sin embargo, esta fórmula padece de grandes errores, y solamente en los últimos años Du Bors y sus colaboradores pudieron calcular en términos precisos el área de superficie. Estos investigadores cubrieron completamente el cuerpo con papeles, los pesaron, los dividieron en diversas figuras geométriias, y calcularon la superficie de cada una de estas figuras dando así la superficie total del organismo. Este procedimiento es conocido con el nombre de Fórmula Linear, y aun se puede aplicar en la infancia hasta los dos años. Años más tarde, estos investigadores simplificaron su procedimiento, y solamente conociendo el peso y estatuta de un individuo es hoy posible calcular el área de superficie. La fórmula usada es la siguiente:

$$
\text { Area cle su. }=\text { Peso } \times 0.425+\text { Estatura } \times 0.725+71.84
$$

dando el árez en centímetros cuadrados, el peso eir kilogramos y la estatura en centímetros. Esta fórmula es precisa entre $5 \%$ más o menos, y sobre ella se han construído diagramas que permiten conocer el área de superficia conociendo los factores peso $y$ estatura.

Sin embargo, la relación entre la producción de calor y el área de superficie no es aceptada por todos los investigadores. HARRIS y BENEDICT se oponen a aceptar este hecho, y dicen quie la ley de Newtax de enfriamiento no puede aplicarse a cuerpo viviente. porque el cambio de producción de calor a diferentes temperaturas del ambiente, no es proporcional a estos cambios de temperatura, puesto que la piel y tejidos subcutáneos, y la distribución de la sangre periférica tienen influencia sobre la cantidad de calor que se pierde, e igualmente han demostrado, que durante los períodos de inanición la disminución en el metabolismo no es proporcional a la disminución del peso y, por consiguiente, tampoco al área de superficie. Sin embargo, esta última objeción no se aplica a individuos normales sujetos a una alimentación suficiente.

En igr7, Aub y Du' Bors hicieron una revisión de la literatura hasta ese año, y teniendo en cuenta las variaciones que sufre el metabolismo según la edad y sexo, publicaron una tabla de valores normales en que dan la producción calórica expresada en calorias por hora y por metro cuadrado de superficie, 
descle los it hasta los 80 años de edad, y expresaron la idea de que el metabolismo de un individuo oscila normalmente entre más o menos $10 \%$ de los valores dadus en eja tabia.

La tabla es la siguiente:

Valores Normales de Aub-DuBors

Calorias por $\mathrm{m}_{2}$ de superficie y por hora

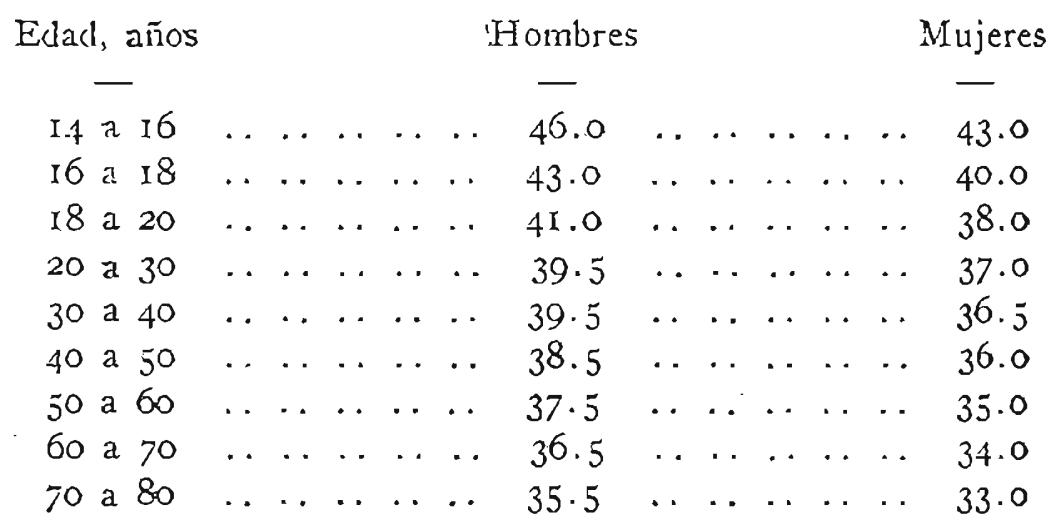

Esta tabla de valores normales ha sido adoptada en todas las clínicas americanas y europeas; actualmente es la usada en el estudio comparativo del metabolismo básico, y es la que establece el valor normal y por consiguiente la desviación metabólica, si es que existe. Boothby y SANDirord, en Estados Unidos, estudiando el metabolismo normal de $12 \gamma$ individuos, encontraron que $92 \%$ tenían un metabolismo oscilando entre los límites normales de más o menos ro\% del valor calculado para su edad, sexo y por $\mathrm{m}_{2}$ de superficie, y que $99 \%$ se encontraban entre los límtes de más y menos $15 \%$.

HaRris y BENEdICt, desconocienclo el valor de referir la producción calórica al área de suverficie, han introducido las siguientes fórmulas para predecir la producción calórica de un individuo en 24 horas. Las fórrrulas san las siguientes:

Para hombres:

Calorias en 24 horas $=66.4730+13.7516$ peso +5.0033 est. 6.755 edad 
Para mujeres:

Calorias en 24 horas $=655.0955+9.5634$ peso $+\mathrm{r} .8496$ est. 4.6756 edad

en que el peso es dado en kilogramos, la estatura en centímetros y la edad en años.

Boothby y SANdiroRd, en una serie de 404 determinaoiones, indican que el promedio de diferencia en el cálculo del metabolismo por medio de los valores de $A u B-D u$ Bors y $\mathrm{HA}_{\mathrm{A}}$ RRIS BENEDICT es solamente de $6.5 \%$, haciendo notar que la discrepancia principal entre los dos métodos está en los valores atribuidos a los factores de edad y sexo. Du Bors cree que el metabolismo disminuye con la edad en igual proporción en individuos grandes y pequeños, mientras que HarRIS y BENEDICT creen que en un sujeto pequeño la disminución del metabolismo con el transcurso de los años es mayor que en uno grande. Igualmente HARRIS y BENEDICT creen en una acción diferente del sexo con respecto al tamaño, afirmando que las mujeres pequeñas tienen una mayor producción calórica que los hombres de igual proporción, mientras que los hombres grandes ticnen una mayor producción calórica que las mujeres igualmente desarrolladas.

Dreyer ha propuesto varias fórmulas para la predicción del metabolismo, y usa como factores la edad, sexo, peso, circunferencia del tórax, longitud del tronco, etc. Sin embargo las fórmulas más usadas en las clínicas americanas y europeas son las de Aub-Du Bois y Harris Benedict.

La aplicabilidad de los valores normales discutidos constituye uno de los objetos principales de nuestro trabajo, y la posibilidad de adaptar en nuestro medio y en distintos lugares las cifras dadas por estos investigadores, para que sirvan de base en el estudio de los desórdenes metabólicos en la clínica; o la posible modificación de estos valores, si nuestra raza indígena posee un nivel metabólico diferente como resultado racial o como influencia de las diferentes condiciones de vida en nuestro país. 


\section{Presentación del Trabajo}

Las determinaciones de metabolismo que a continuación presentamos, han sido hechas usando los aparatos de SANBorn, perteneciente a la Facultad de :Medicina, y de Benedict-Rota, propiedad de la Sanidad Militar, aparatos en cuyo manejo adquirimos suficiente preparación como Médico Interno del Boston Hospital (Boston, EE. U.U.), a cargo del laboratorio de Metabolimetría.

Todas las determinaciones han sido practicadas por medio de la calorimetría indirecta, calculando el consumo de oxígeno en un período dado (seis minutos) y usando el método gráfico de registro de respiraciones y medida diel oxígeno, método que permite apreciar inmediatamente la clase de cooperación del individuo metabolizado. Antes de proceder a una determinación, se explicaba cuidadosamente al sujeto que iba a ser metabolizado el significado de la prueba y la falta de peligro en la determinación y se le familiarizaba con el uso del aparato, haciéndolo respirar oxígeno por un tiempo corto. A pesar de estas precauciones, muchos resultados han sido desechados y no considerados en este trabajo, por presentar una respiración irregular o por nerviosidad excesiva.

El oxígeno consumido fué adquirido en la fábrica de este gas en Lima, y transportado en cilindros a los diversos lugares de estudio. La cal sodada, usada en los aparatos para absorción del anhídrido carbónico, fué encargada a Estados U/nidos, llevando la etiqueta de "Wilson Soda Lime".' Los aparatos eran probados frecuentemente para evitar escape de gas.

Todos los sujetos metabolizados se encontraban en ayunas, practicándose la determinación en la mañana, después de reposar a lo menos media hora y casi sin actividad física en las horas antes de la determinación. Inmediatamente después de ésta, eran pesados y medidos, y el pulso era determinado antes de ser conectados con el aparato.

El área de superficie ha sido calculada por medio de las Tablas de Aub-Du Bors, y los resultados comparados con los valores normales expresados por Aub-Du BoIs y Benedict, siendo corregidos previamente por temperatura y presión barométrica. 
Las determinaciones en Lima, Arequipa, Santa Lucía y Piura, han sido hechas personalmente, y las verificadas en la Oroya, por el doctor Enrique Rondón bajo nuestra inmediata dirección, durante la expedición científica enviada por la Facultad de Medicina para investigar la vida en las alturas.

\section{Estudios de Metabolismo básico en Lima (I)}

En las primeras páginas de este trabajo hemos dicho que los valores normales que hoy tenemos en Metabolimetría, acerca de la producción calórica de un individduo, son principalmente los establecidos por Aub-Du BoIs y por Harris-Benedict. Estos valores han sido adoptados en todas las clínicas americana y europeas, aceptando variaciones entre más y menos $10 \%$ como limites de la normalidad.

Pero si estos valores son aplicables a otras razas y a otros centros, y si individuos de caracteres raciales y hábitos de vida completamente distintos poseen idéntica producción calórica, indudablemente que no se ha establecido de una manera precisa; la literatura en este sentido es bastante precaria y no encontramos una información abundante. Los estudios más completos se han hecho entre los japoneses, que bien sabemos poseen caracteres raciales completamente diferentes, con hábitos de vida distintos, incluyendo una dietética diferente, y aun sus caracteres antropométricos difieren notablemente del individuo europeo o americano, siendo de corta estatura y de peso más bajo.

(1) Lima se encuentra en una región subtropical, a latitud 13 sur. Investigaciones en el observatorio de la Facuitad de Ciencias, dan por 11 años un promedlo de:

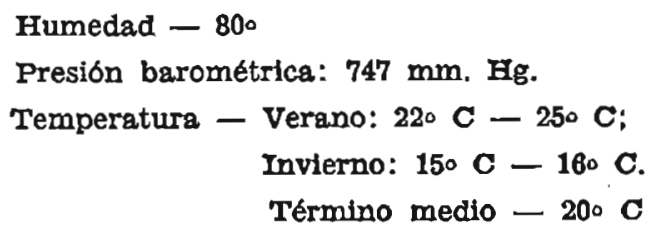


Okada, Sakurai, Kaimeda, Takahira y otros han estudiado el metabolismo de japoneses jóvenes normales, y han encontrado fidénticos valores comparando con los de chinicas europeas y aplicables los valores normales que hemos descrito. Estos investigadores, considerando que la dietética diferente de los japoneses pudiera tener alguna influencia en el metabolismo básico, ya que sus comidas consisten esencialmente en carbohidratos, sometieron a algunos a una dieta diferente y sin embargo este cambio no afectó el metabolismo.

EIJkMAN, en I896, trabajando en Batavia con un aparato Zuntz-GEPPERT, estudió doce sirvientes malayos y once europeos, y encontró idéntica producción calórica al comparar con: determinaciones hechas en Alemania.

Sin embargo, algunos investigadores han encontrado diferencias que bien pueden clasificarse como características metabólicas raciales, importantes de tomar en cuenta. Flem Ming, en I923, determinó el metabolismo de cinco filipinos y halló que todos se encontraban a un nivel metabólico más bajo que el va-lor normal que les correspondía según la escala de AUb-Du Bors, siendo el promedio de desviación de $5.3 \%$. Mc Creod, Crofts y Benedict, estudiaron el metabolismo de nueve mujeres (siete chinas y dos japonesas) de 21 a 29 años de edad, y hallaron igualmente una producción calórica reducida, siendo el término medio de $10 \%$ menos que los valores calculados según la fórmula de Harris-Benedict.

TURNer, en 1926, estudia el metabolismo en Siria, tomando individuos de diferentes razas (egipcios, armenios, persas, etc.), y encuentra que las variaciones de los Standards de $\mathrm{H}_{\mathrm{A}-\text { - }}$ RRIS-BENEDICT no son grandes, pero que había variaciones aunque pequeñas en los diferentes grupos de individuns según la raza, y cree que en el metabolismo hay un factor racial evidente que debe ser investigado.

Del estudio de la literatura vemos, pues, que hay una falta de uniformidad en las diferentes investigaciones, y que si bien parece que hay un factor racial en el metabolismo, éste no se encuentra presente en todas las razas. Este hecho presta más importancia al estudio emprendido, que significa la investigación de los caracteres metabólicos de nuestra raza blanca e indígena. 
En primer lugar damos el resultado de la determinación de metabnlismo básico en un grupo de médicos y estudiantes en Lima, todos de raza blanca, con hábitos de vida bastante diferentes, pues algunos llevan una vida de regular actividad física, y otros sedentaria y poco activa. Los resultados de estas determinaciones se encuentran en la Tabla I, que sigue. 


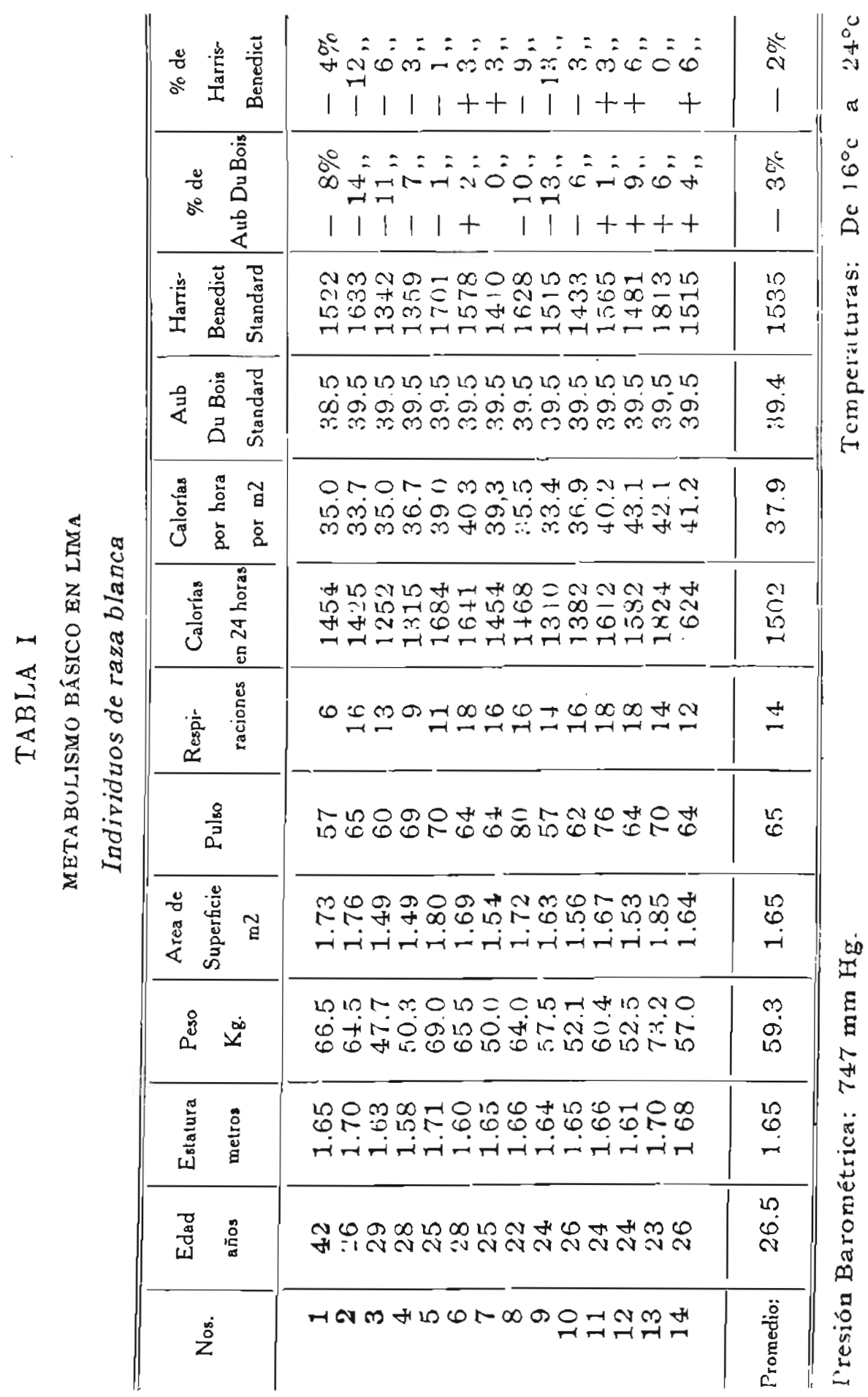


Vemos que la edad de estos individuos varía entre los $22 \mathrm{y}$ 42 años, siendo el promedio general 26 años. Aplicando los valores normales dados por AUB-Du Bors, fencontramos que las desviaciones en los resultados varían entre - $14 \%$ y $+9 \%$ siendo el término medio de $2 \%$. Aplicando los valores dados por HARRIS BENEDICT, las desnfiaciones je encuentran kentre - $3 \%$ y $+6 \%$, siendo el término medio de $2 \%$. Es notable la equivalencia casi completa entre las fórmulas establecidas por estos investigadores, siendo las vàtiaciones y término medio casi 10 mismo. Estós individuos, de raza blanca y de hábitos de vida diferentes, constituyen el tipo de individuo que encontramos comunmente en la práctica diaria, y es importante conocer de este estudio, que podemos aplicar los valores normales establecidos en clínicas americanas, como base comparativa, cambiando un poco los límites normales de variación. $Y$ es interesante considerar que estos hombres de raza latina, de estatura y peso menor que los americanos y de una vida sedentaria, poseen el mismo nivel metabólico. La dietética en estos lugares, comparando con otros centros, es quizás más rica en carbohidratos y grasas y pobre en proteínas, pero ya hemos visto cómo la dietética diferente de los japoneses no influye en el nivel metabólico, y BENEDrCT analizando este punto halló que no hay diferencia en el metabolismo de los vegetarianos y de los no vegetarianos. En seguida damos, en la Tabla II, el primer estudio metabólico de un grupo de individuos de raza indígena, todos provenientes de la sierra (generalmente de altos lugares), con buena salud y cuya estadía en Lima varía desde dos semanas hasta cuatro años. El grupo estudiado pertenece al ejército, y, por consiguiente, lleva una vida bastante uniforme, con una dieta rica en carbohidratos suficiente para llenar sus necesidades calóricas. 


\begin{tabular}{|c|c|c|c|}
\hline & 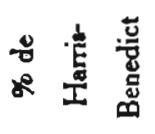 & 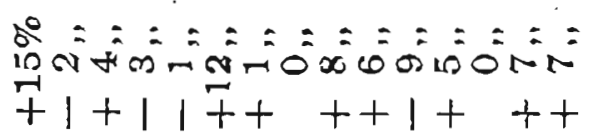 & $\begin{array}{l}\text { \&u } \\
+\end{array}$ \\
\hline & 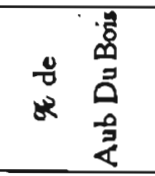 & 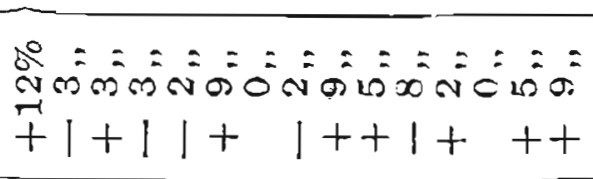 & $\begin{array}{l}\stackrel{2}{\circ} \\
+\end{array}$ \\
\hline & 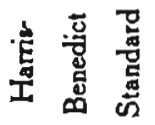 & 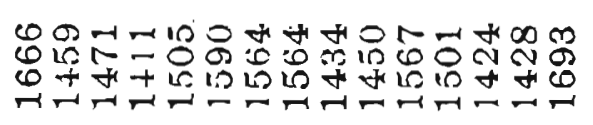 & $\begin{array}{l}\infty \\
0 \\
0 \\
2\end{array}$ \\
\hline & 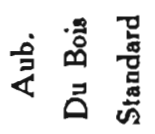 & 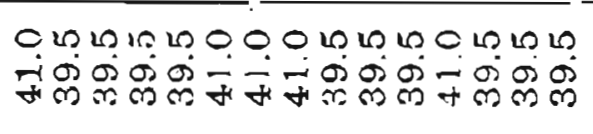 & $\stackrel{0}{\dot{4}}$ \\
\hline 或喿 & 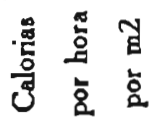 & 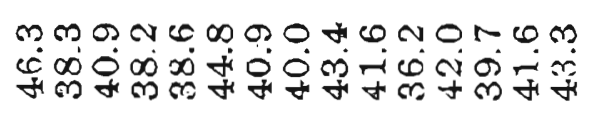 & $\stackrel{0}{4}$ \\
\hline 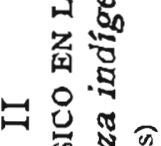 & औึ & 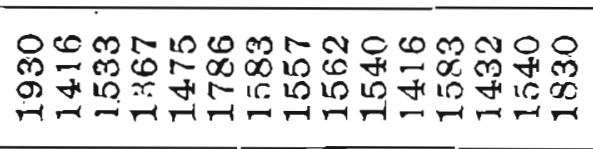 & $\stackrel{8}{\text { ris }}$ \\
\hline 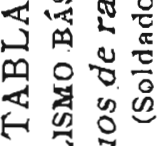 & 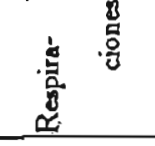 & 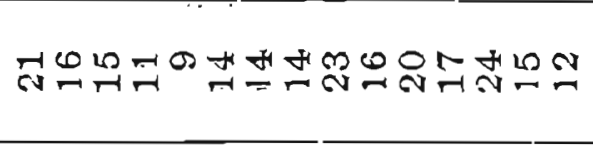 & $\stackrel{\varphi}{\sim}$ \\
\hline$\sum_{0}^{0} \cdot \frac{0}{6}$ & $\frac{0}{3}$ & 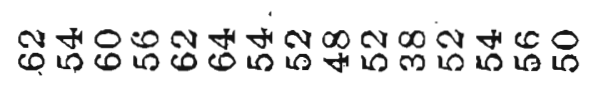 & हैं \\
\hline & 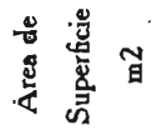 & 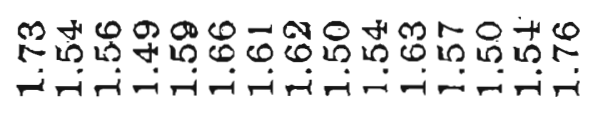 & ن \\
\hline & $\stackrel{8}{0} \not$ & 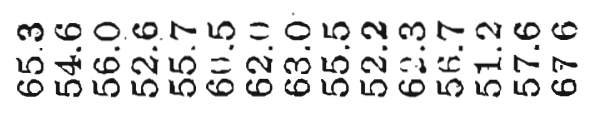 & $\infty$ \\
\hline & 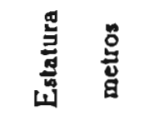 & 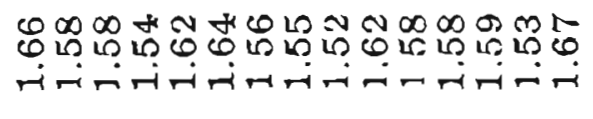 & - \\
\hline & 莺 总 & 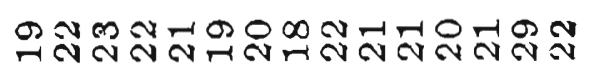 & $\vec{o}$ \\
\hline & $\sum^{\circ}$ & HNm & : \\
\hline
\end{tabular}


El resultado de estas determinaciones, expresadas en la Tabla II, es muy interesante, pues encontramos que el metabolismo básico de estos indígenas cae entre los mismos límites que el grupo anterior, y por consiguiente es posible aplicar los valores normales de Aub-Du Bors y Harris Benedict. Según la primera escala, las variaciones de metabolismo en este grupo varian desde $-8 \%$ hasta $+\mathrm{I} 2 \%$, siendo el promedio general de $+2 \%$, y según la fórmula de HarRis-BENedict las variaciones son desde $-9 \%$ hasta $+15 \%$ con un término medio de $+4 \%$. Es, pues, notable aquí igualmente la uniformidad en los resultados de la aplicación de estas dos fórmulas.

Los resultados de estas determinaciones son los primeros datos que tenemos acerca de la aplicabilidad de los valores normales usados en otros centros; tenemos que recordar, que estos son los enfermos que encontramos en nuestros Hospitales, y, por consiguiente, el establecimiento de estas bases normales posee singular importancia y nos indica que el indio peruano al bajar de sus montañas y campos para habitar en las ciudades civilizadas, adaptándose a nuestros hábitos de vida, posee el mismo nivel metabólico de los hombres blancos y de otras razas con caracteres antropométricos tan diferentes, ya que hemos de recordar la baja estatura y alto peso de estos indígenas y, lo que es más interesante, el tórax amplio y enfisematoso, con una capacidad vital bastante elevada. Es interesante a este respecto ver que el número de pulsaciones de estos individuos es de $\mathbf{5 4}$ por minuto, y en cambio en los individuos de raza blanca el término medio es de 65 , a pesar de un metabolismo algo más bajo; no obstante, de otros estudios sabemos que la correlación del pulso y del metabolismo es un fenómeno inctividual es decir, en un individuo dado a un aumento de metabolismo corresponde generalmente un aumento de pulso; pero a un nivel metabólico normal no corresponde determinado número de pulsaciones.

Tratando de establecer sobre bases más firmes el hecho de que a los individuos de raza indígena en Lima son aplicables los valores normales que hemos discutido, fué emprendido el estudio del metabolismo básico de un grupo de mujeres indígenas, igualmente provenientes de lugares altos de la Sierra, y cuya estadía en Lima varía desde uno hasta ocho años, encontrándose todas en buen estado de salud. 


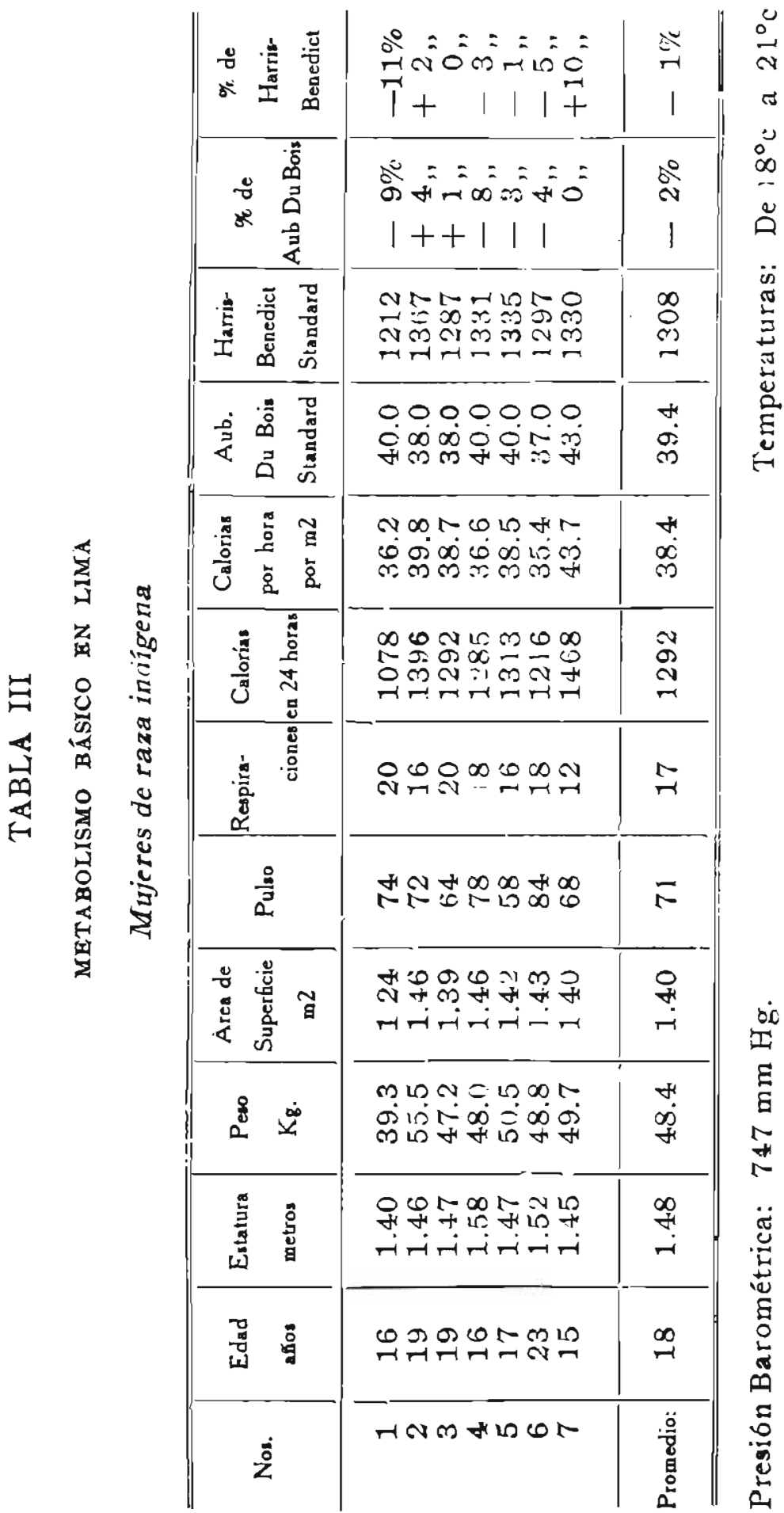


Según los resultados expresados en la Tabla III, vemos que estas mujeres poseen igual nivel metabólico báspico que los grupos estudiados anteriormente. Ségún la escala de AuBDu Bors, las variaciones se encuentran entre $-9 \%$ y $+4 \%$, siendo el término medio de $-2 \%$; y según la fórmula de $\mathrm{HA}_{\mathrm{A}}$ RRIS-BENEDICT, los metabolismos oscilan entre -Ir\% y + IO $\%$, siendo el primedio general de - I \% L Los resultados de estas investigaciones confirman, pues, el hecho de que estos individuos de raza indígena poseen un metabolismo igual al de otras razas en Lima.

Las anteriores determinaciones nos permiten, pues, afirmar el hecho de que en Lima son aplicables los valores establecidos en otras clínicas como producción calórica normal, apesar de la diferencia en razas y costumbres y que sólo sería conveniente ampliar algo más los límites de variaciones normales.

\section{Estudios de Metabolismo Básico en las alturas}

La vida en las alturas presenta serios inconvenientes, a los cuales el organismo humano tiene que adaptarse mediante ciertos fenómenos fisiológicos de compensación, fenómenos que hoy se encuentran en pleno estudio. En estos aitos lugares la baja tensión atmosférica dificulta la propia oxigenación de la sangre, y en consecuencia el organismo sufre ciertos trastornos, especialmente en el individuo cuya ascensión ha sido rápida y que se encuentra imposibilitado de ejercer una regular actividad física sin sufrir los efectos del "soroche", o sea el mal de las alturas.

El organismo al adaptarse a esta vida en las alturas presenta ciertos procesos interesantes, tales como el aumento de gló. bulos rojos, la mayor capacidad torácica, la dilatación capilar, etc., y como sabemos que cualquier actividad física es seguida de un fuerte desgaste de energía, que impide a estos individuos entrar de lleno en la vida ordinaria, es interesante averiguar si igualmente existen fenómenos de adaptación metabólica, es decir si el organismo se acomoda a esta nueva vida con un nuevo nivel metabólico y si hay una diferencia en la actividad íntima celular del individuo o nativo de estos lugares, comparado con él que 
principia a adaptarse o sufre de los síntamas cle la falta de oxigenación necesaria.

Los previos estudios a este respecto parecen indicar que no existen estos supuestos fenómenos de adaptación metabólica. En 1902, VoN SChrotTer y ZuNTZ practicaron una ascensión en globo a las alturas de 4560 y 5160 metros, y determinando el metaluolismo ha:laron en uno de ellos un aumento de $7 \%$ en el consumo de oxígeno, pero en el otro el consumo fué menor, atribuyendo el aumento en el primero al mayor trabajo de los músculos respiratorios.

En I904, Duric y Zuntz ascendieron al Col d'Olen a una altura de 2900 metros, y durante una semana que vivieron en esta altura no encontraton alteración en el metabolismo, ni aumento de la energía necesaria para practicar algún trabajo. Después ascendieron al Monte Rosa, a 4560 metros de altura y a una presión barométrica de $443 \mathrm{~mm}$., y hallaron un aumento en el metabolismo de $15 \%$, e igualmente una mayor liberación de energía para ejlercer cualquier actividad física.

Douglas, Haldane, Henderson y Schneider, en igr 3 , durante la ascensión al Pike's Peak, no encontraron variación en el metabolismo básico, pero éste fué determinado solamente después de estar aclimatados. En 1915, Hasselbach y Lindhard sometieron a varios individuos a una baja tensión de oxígeno en cámaras respiratorias, igualando así las condiciones fisiológicas que se encuentran en las alturas, y tampoco hallaron cambio alguno en el metabolismo básico. SCHNEIDER, en 1923, observó un individuo en la altura cuyo metabolismo fué de I $5 \%$ inás alto ante de aclimatarse. En Ig22, Barcroft, Binger, Bock, Doggart, Forbes, Harrop, Meakins y Redfield, durante la expedición al Cerro de Pasco, no encontraron alteraciones en el metabolismo, pero sí evidenciaron un mayor número de pulsaciones en estas alturas.

Si analizamos todos estos previos estudios, encontramos que la' gran mayoría de las investigaciones se han llevado a cabo casi. inmediatamente despliés de la ascensión a estos altos lugares, y no se ha hecho distinción alguna en el estudio del metabolismo de aquellos individuos que se adaptan inmediatamente a la nueva vida, comparándolos con aquellos que sufren del mal de las alturas. Tampoco se ha irivestigado el metabolismo básico de los 
nativos ni de los residentes por largos años en estos altos lugares y por consiguiente perfectamente adaptados.

En primer lugar estudiaremos el metabolismo básico de un grupo de individuos (médicos y estudiantes) de raza blanca, cuyo metabolismo en Lima, al nivel del mar, hemos visto que variaba entre límites normales de la escala de AUB-Du Bors y la fórmula de HARris-Benedict, y que ascendieron en un sólo día a la Oroya, por medio del ferrocarril, lugar a r2000 pies de altura, a una presión atmosférica de $480 \mathrm{~mm}$., con una temperatura que oscilaba entre los $12^{\circ} \mathrm{C}$. y $17^{\circ} \mathrm{C}$., siendo el metabolismo determinado durante los primeros diez días de llegar a este lugar. Es de advertir que todas estas determinaciones fueron practicadas en lugares completamente adecuados, es decir en habitaciones abrigadas, y con todas las comodidades necesarias para evitar cualquier factor de incomodidad o inclemencia del tiempo.

En el estudio del metabolismo de este grupo, creímos conveniente separar aqıellos que sufrían de los sintomas del soroche, y cuya vida en las alturas presentaba una evidente falta de adaptación llegando a pasar momentos bastante críticos durante la ascensión, comparando el estudio de estos casos patológicos con aquellos que ascendiendo a estas alturas, no presentaron ninguna perturbación evidente, y que pudieron seguir una vida normal, aunque naturalmente algo restringida en su actividad física. En la Tabla IV, que sigue, damos el resultado de estas determinaciones. 


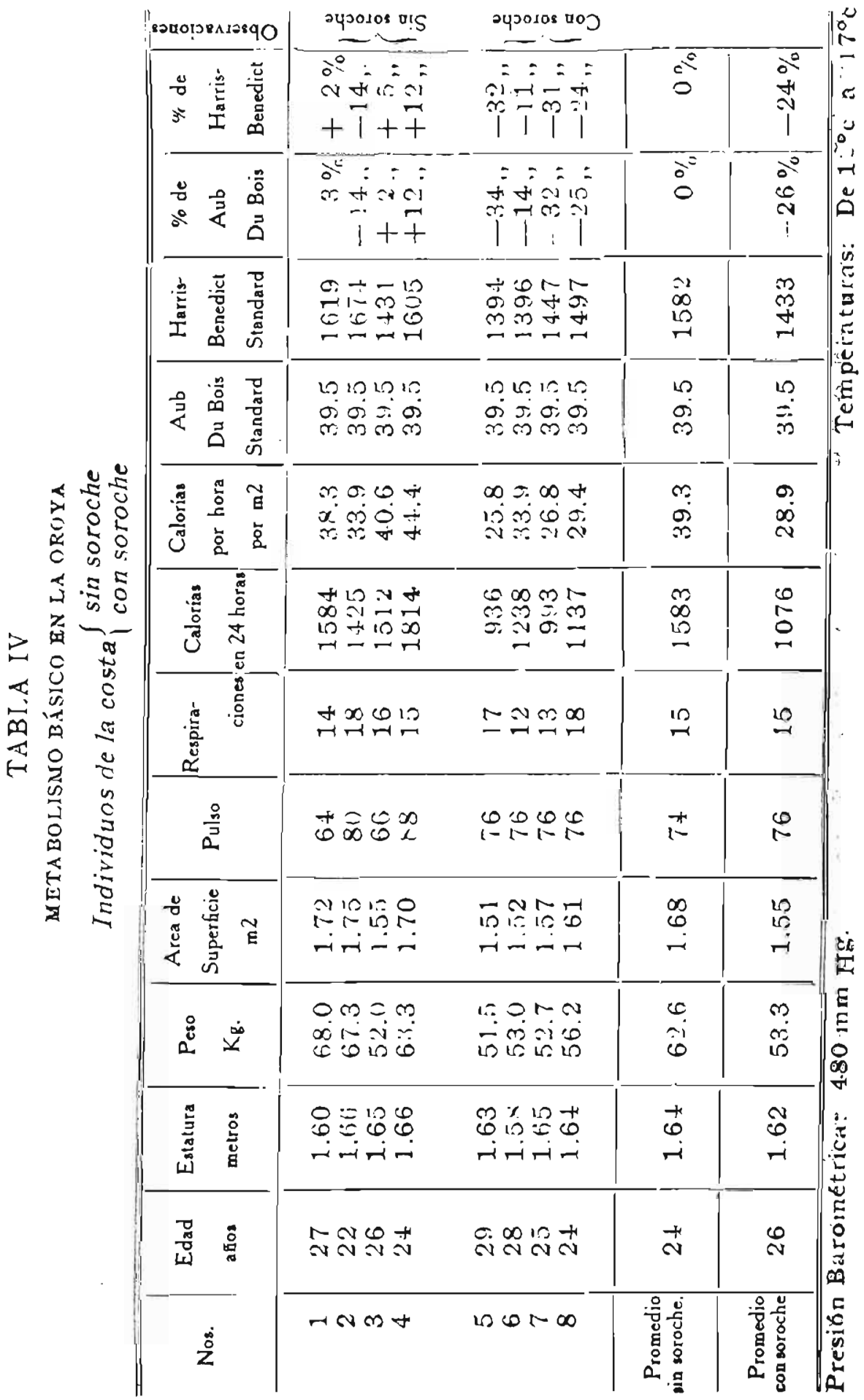


Los resultados de estas determinaciones dadas en la Tabla IV son en extremo interesantes. A primera vista nos damos cuenta de que aquellos individuos que sufrieron del "soroche", - sea la falta de adaptación a la vida en las alturas, exhibieron un metabolismo mucho menor, en límites completamente anormales (de $-\mathrm{I} 4 \%$ a $-34 \%$ ), comparados con aquellos cuya nueva vida en las alturas no causó ninguna perturbación aparente, es decir, con los que se adaptaron inmediatamente y cuyo metabolismo se encontraba entre límites normales (-i4\% y $+\mathrm{I} 2 \%)$. Es de advertir que de los cuatro que sufrieron del soroche, los que experimentaron síntomas más agudos mostraron una mayor reducción en su producción calórica.

La explicaoión de estas interesantes determinciones es quizás algo dificil, y los actuales estudios no permiten la explicación de este fenómeno, señalado por vez primera sino en términos teóricos. Hemos visto que cualquier actividad física en estos altos lugares es seguida por un desgaste de energía considerable, y que el número de pulsaciones es mayor evidenciando un aumento de trabajo cardíaco; es lógico suponer que este desgaste de energía y calor, aun presente en los individuos adaptados en las alturas, sea pues mayor, y cause una perturbación más seria en los sujetos no adaptados, que bien sabemos experimentan una serie de síntomas agudos al menor ejercicio, y por consiguiente podemos suponer que esta disminución de metabolismo básico en este último grupo del soroche, puteda ser clasificada como un fenómeno compensatorio, significando que cuando uno de estos individuos se enfrente a cualquier actividad física, el organismo se encuentre a un nival más bajo de actividad celular, y por consiguiente más apto para soportar la pérdida calórica y de energía que esta actividad le cause. Quizás esta explicación sea la más lógica, pero es evidente que futuros estudios son necesarios para demostrar la veracidad de esta teoría que adelantamos. Un hecho, que soporta bastante esta teoría de economía calórica, lo encontramos en un experimento verificado meses más tarde en Santa Lucía, a 4038 metros sobre el nivel del mar y a una presión atmosférica de $464 \mathrm{~mm}$. En este lugar determinamos en idénticas condicions el metabolismo básico de un individıo nativo de esas alturas y a quien el más violerto ejercicio no afectaba en lo más mínimo, por consiguiente adaptado perfectamente a las condiciones fisiológicas de 
la altura, e igualmente el metabolismo básico de un indjviduo de la costa, recién ascendido a esos lugares, y que a pesar de sentirse perfectamente bien le era imposible desarrollar la misma actividad física del sujeto anterior, sin sentir los efectos de la falta de adaptación. En seguida estos clos individuos eran sometidos al mismo ejercicio, exactanente durante el mismo tiempo, y el metabolismo calculado inmediatamente y quince minutos después de estar en clescanso. En la Tabla $V$ damos los resultados de este experimento. 


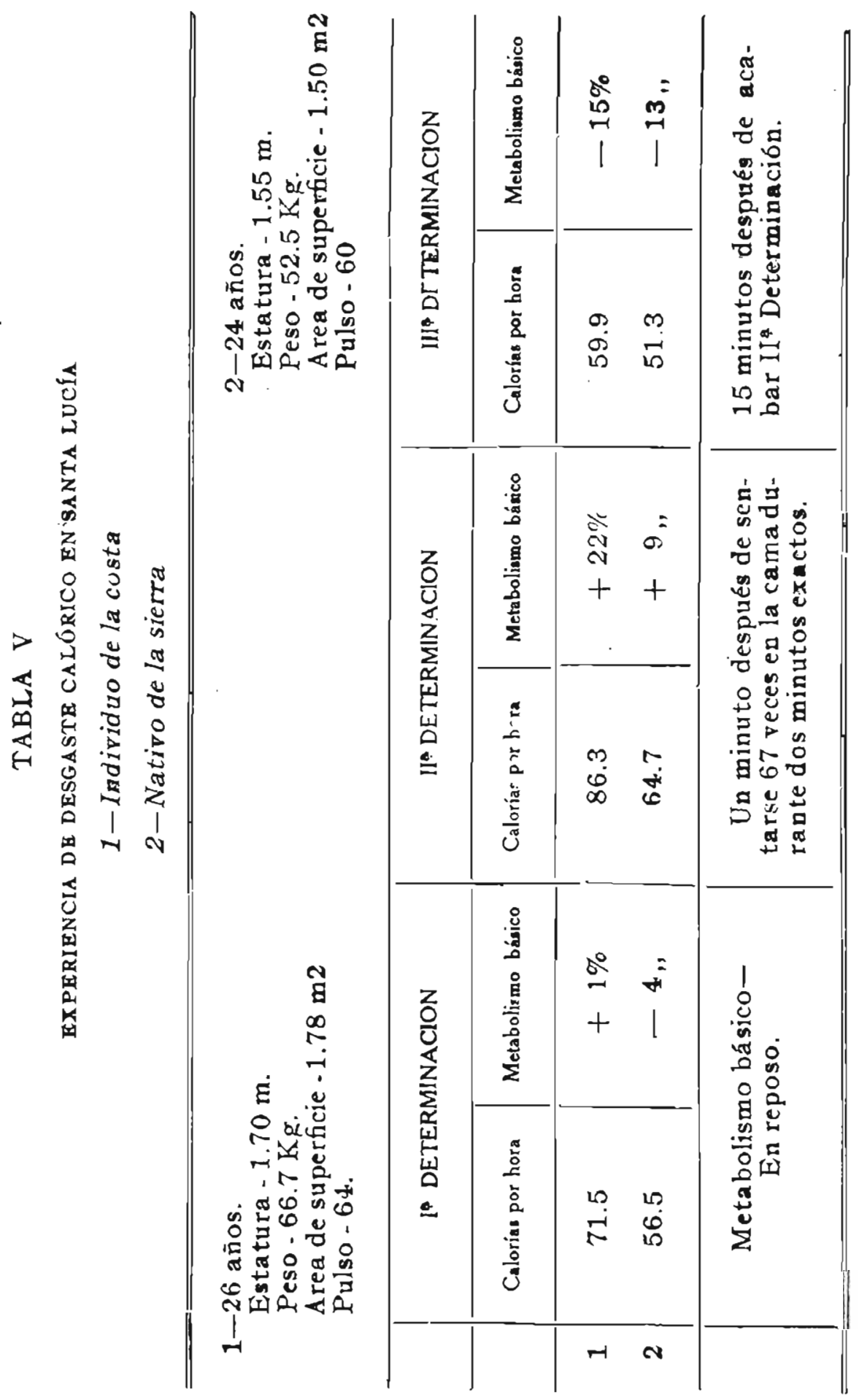


Los resultados. de este experimento son iglialmente muy interesantes. Vemos que en el individuo costeño, sometido al mismo ejercicio que e! nativo de estos lugares, hay un mayor desgaste calórico y un mayor aumento de metabolismo, evidenciando así que uno de los fenómenos de adaptación a estos altos lugares, es un menor gasto de energía al practicar cualquier ejercicio, y por consiguiente el organismo sufre menos, y pliede practicar actos que el no adaptado está imposibilitado de hacer, porque su nivel metabólics sube a limites muy altos ocasionando una perturbación metalsólica evidente. Es asimismo interesante observar, que después del período de descanso hay un descenso del metabolismo básico a límites aun más bajos que al iniciar el experimento, y quizá esta disminución igualmente representa un fenómeno compensatorio del organismo, que disminuye su actividad metabólica, para compensar el gasto calórico anterior, ya que en estos altos lugares la economía en el consumo de oxigeno es necesaria. Los resultados de este experimento pueden apreciarse mejor en el diagrama que sigue: 


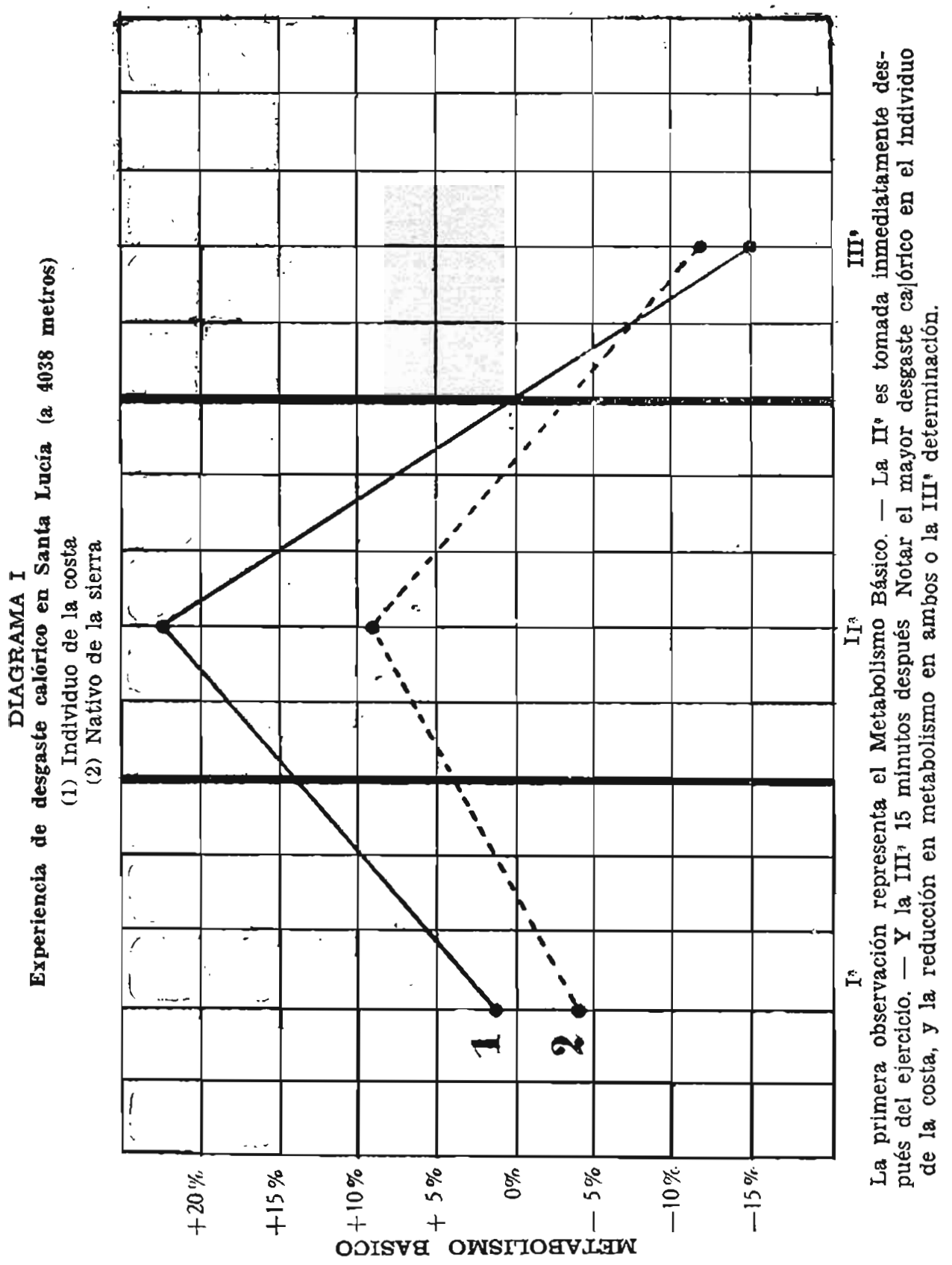


Hemos visto que el metabolismo básico de individuos provenientes de lugares más bajos y que al ascender a estas alturas no exhiben sintomas de inadaptación, se encuentra entre límites normales. En seguida damos el resultado de la determinación del metabolismo en la Oroya de un grupo de nativos de estas regiones, y de residentes por largos años (todos indigenas), que vivian perfectamente bien y en completa adaptación. Los resultados de esta investigación se encuentran en la Tabla VI. 
FACULTAD DE MIFDICINA

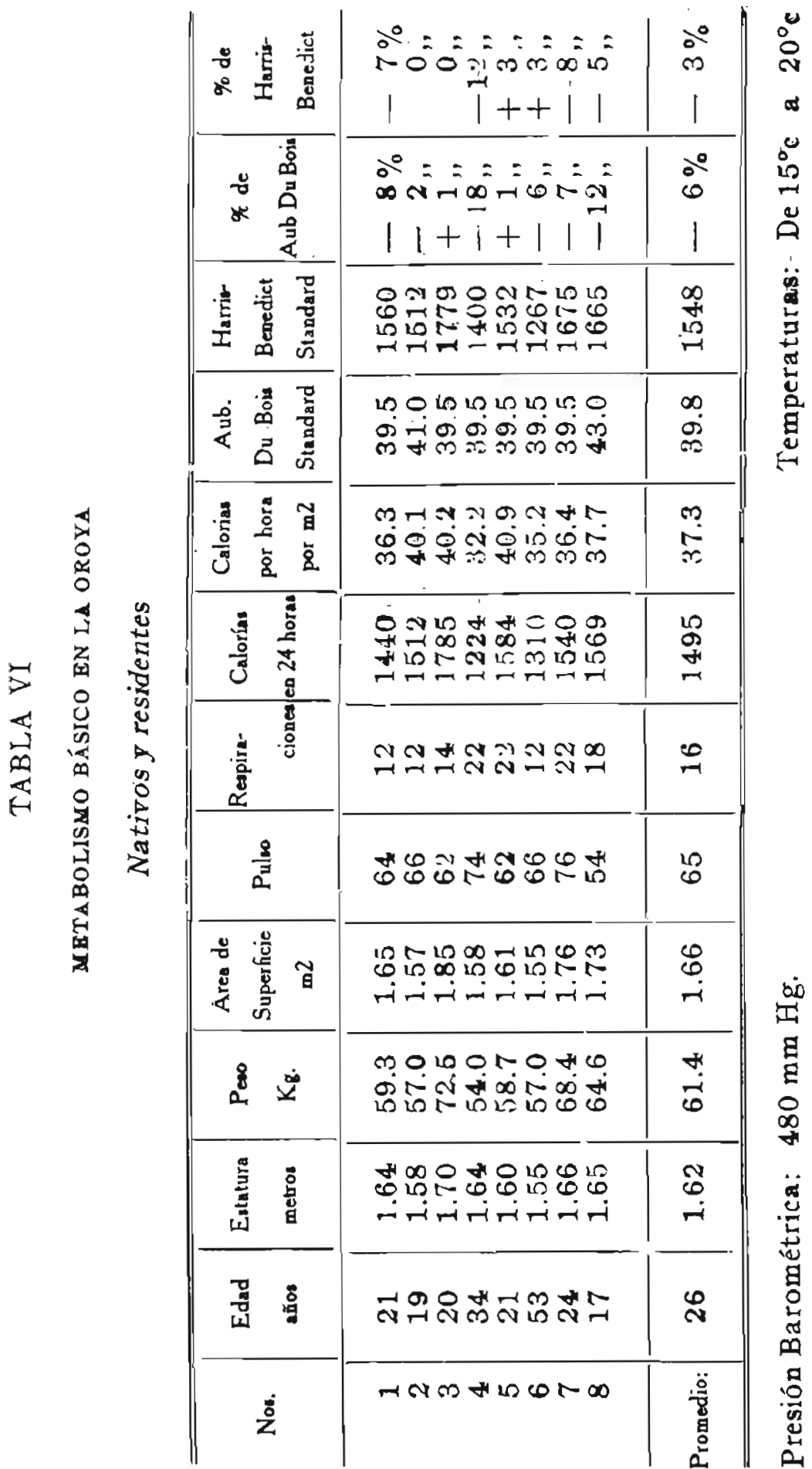


Vemos que el metabolismo básico de estos individuos adaptados, y con larga residencia en la Oroya se encuentra entre límites normales. El metabolismo básico de los individuos que llevan una vida normal en las alturas no parece, pues, exhibir alteraciones importantes. Es interesante notar que el númerø de pulsaciones por minuto es mayor, pues como recordamos al determinar el metabolismo de un grupo de indigenas en Lima, el promedio de pulsaciones era de 54 y aquí asciende a 65 , heche que confirma el mismo hállazgo de previos investigadores y que evidencia in plano mayor de actividad cardíaca.

Para fortuna nuestra tuvimos oportunidad de estudiar en este mismo sitio de la Oroya un grupo de soldados de raza incligena, provenientes de luglares más bajos, eln muchos de ios cuales se notaban sintomas de fatiga e inadaptación, y que, por consiguiente, presentaban una situación análoga, aunque menos aguda, que los indivicluos de la costa, que al ascender caían víctimas del soroche y en los cuales hemos demostrado una disminución de metabolismo básico. Los resultados de estas determinaciones los damos en la Tabla VII. 


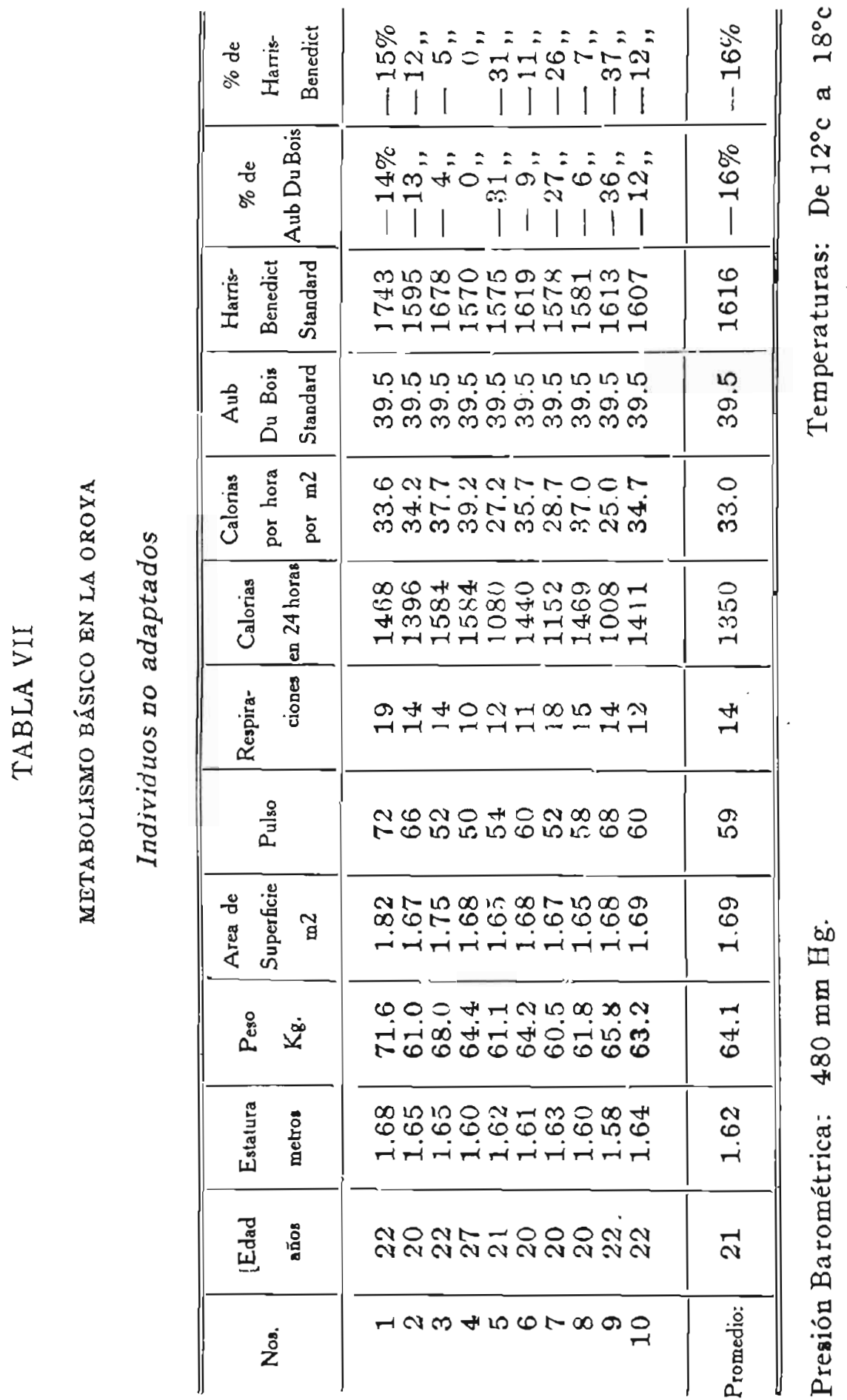


Encontramos que el metabolismo básico de este grupo de indigenas, algunos de los cuales presentaban sintomas de inadaptación, cae en límites bastante bajos y aun completamente patológicos, comparándolos con los indigenas libres de síntomas y nativos de estos lugares, confirmando así nuestra afirmación de que en estos individuos no adaptados, la disminución de metabolimo básico, que frecuentemente se encuentra; forma parte de uno de los tantos fenómenos compensatorios del organismo y constituye una actividad orgánica más reducida para soportar mejor el desgaste calórico que cualquier actividad causa. Es interesante notar que el número de pulsaciones por minuto es menor en este grupo, que podemos llamar patológico, que en el de individuos normales.

Las anteriores observaciones de la no variación en el metabolismo básico de los individuos provenientes de la costa y que no sufrian sintomas de inadaptación en estos altos lugares, pudo ser confirmada igualmente meses más tarde en Santa Lucía ( 4038 metrus de altura y a una presión barométrica de $464 \mathrm{~mm}$ ), determinado el metabolismo básico de dos médicos a los pocos días de llegar a estos lugares, cuando no presentaban síntoma alguno. Los resultados de estas determinaciones se expresan en la Tabla VIII. 


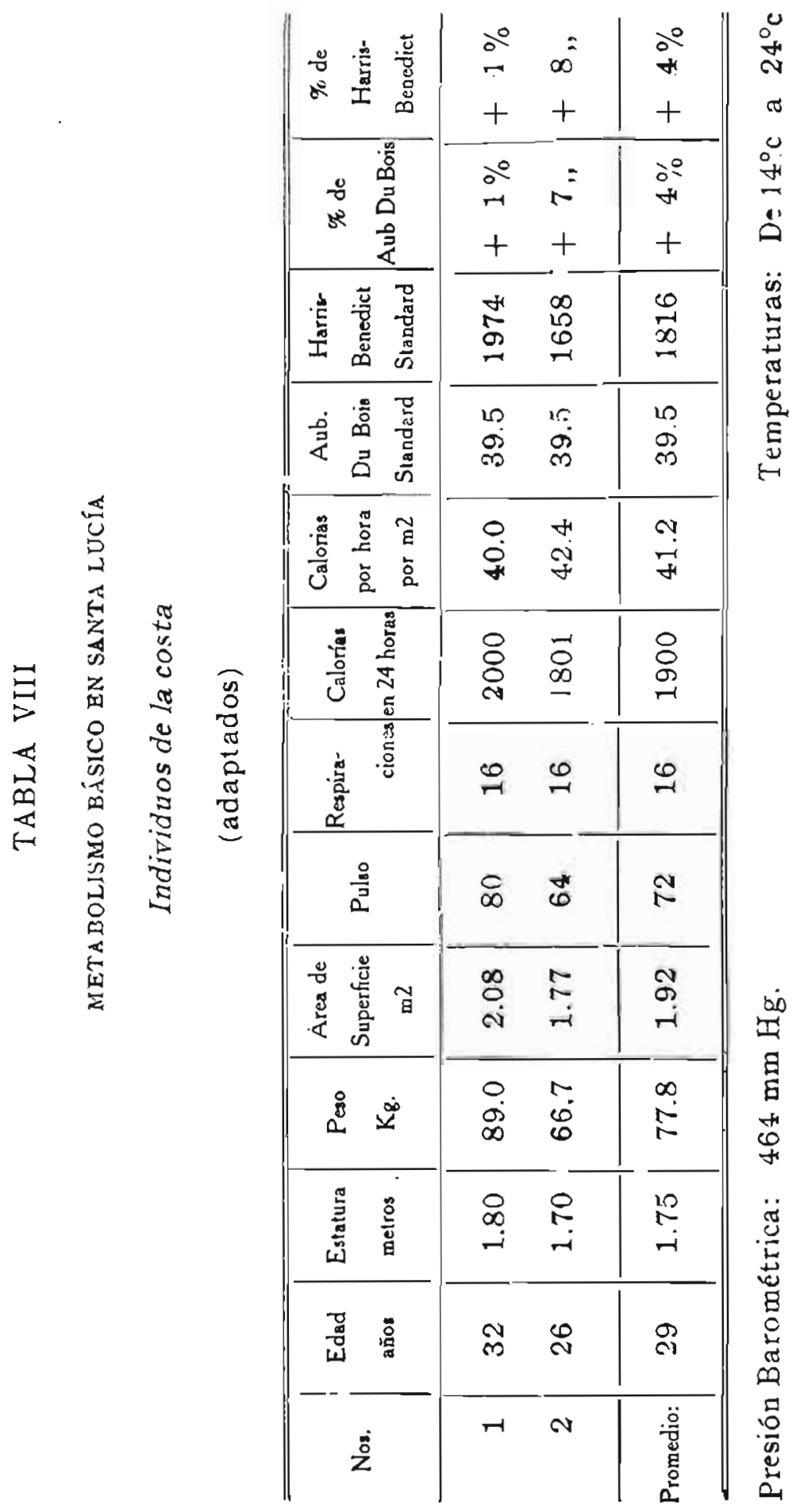


Vemos que el resultado de estas determinaciones se encuentran cntre límites completamente normales. Igualmente la normalidad del metabolismo de individuos que en estos altos lugares no experimentan sintoma alguno, la encontramos en los resuitados de las determinaciones expresadas en la Tabla I,X, en la que presentamos el estudio metabólico de un grupo de soldados indigenas provenientes de diferentes lugares de la Sierra, y que durante la permanencia de unas cuantas semanas en Santa Lucía (a 4038 metros de altura) se hallaban perfectamente bien y pudieron tomar parte en las maniobras militares sin experimentar fatiga alguna, encontrándose, piıes, completamente adaptados. Los resultados de estas determinaciones se presentan en la Tabla IX. 


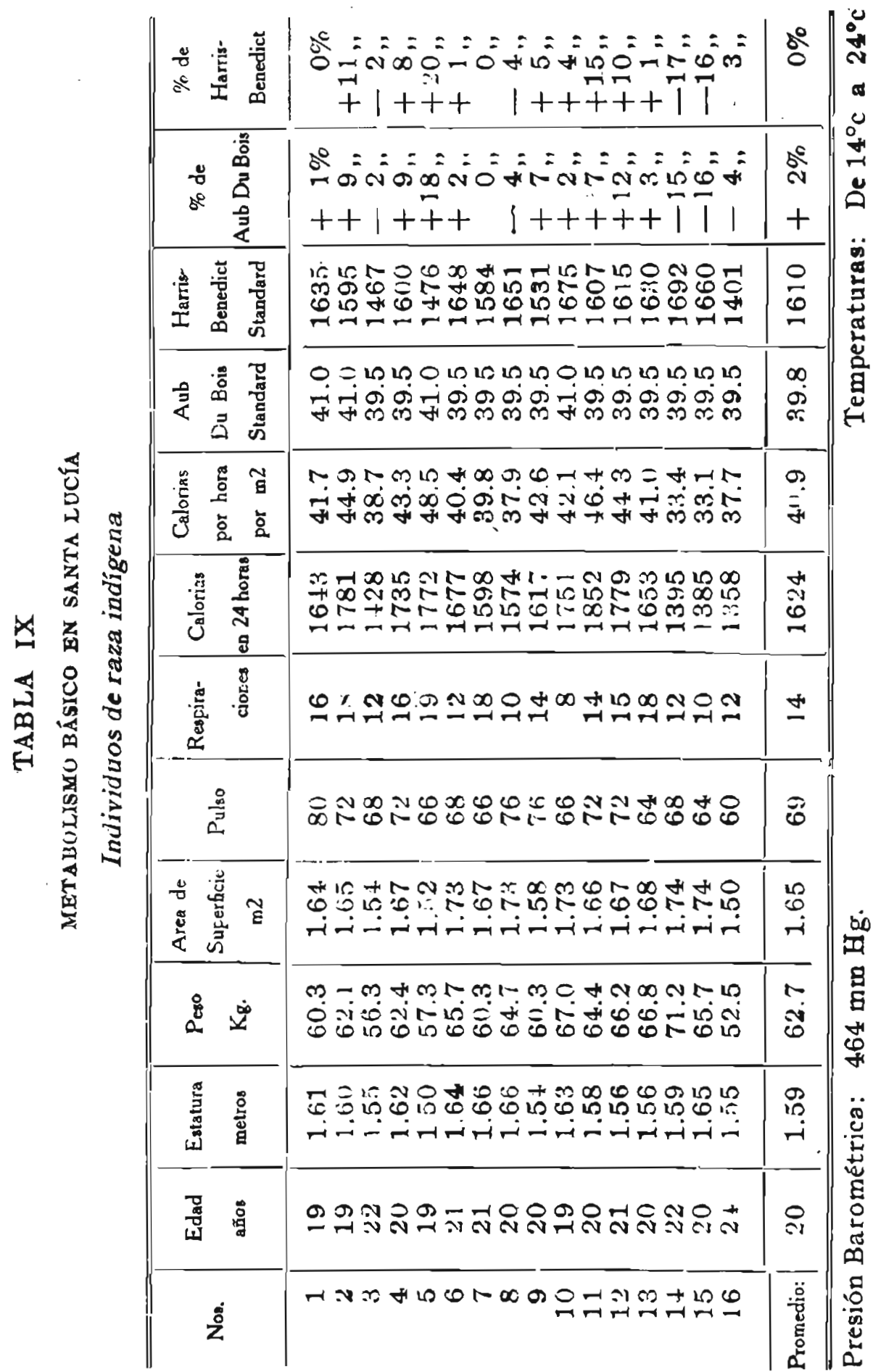


Vemos, pues, que el metabolismo básico de este grupo de indigenas, perfectamente normales, adaptados a estos altos lugares y capaces de soportar una vida de actividad física considerable, se encuentra entre límites completanente normales. Los vilores altos obtcnidos en algumos casos, quizás se explican por la vida activísima de estos sujetos ell maniobras, ya que sabemos por otros investigadores que una vida de actividad física coniderable cansa un aumento en el netabolismo básico.

En Santa Lucía tuvimos oportunidad de practicar determinaciones de metabolismó básico en un grupo de mujeres, todas nativas de estos lugares-por consiguiente perfectamente adaptadas-y que representan el metabolismo básico de nativas. En la Tabla $\mathrm{X}$ damos el resultado de estas determinaciones. 
FACULTAD DE MEDICINA

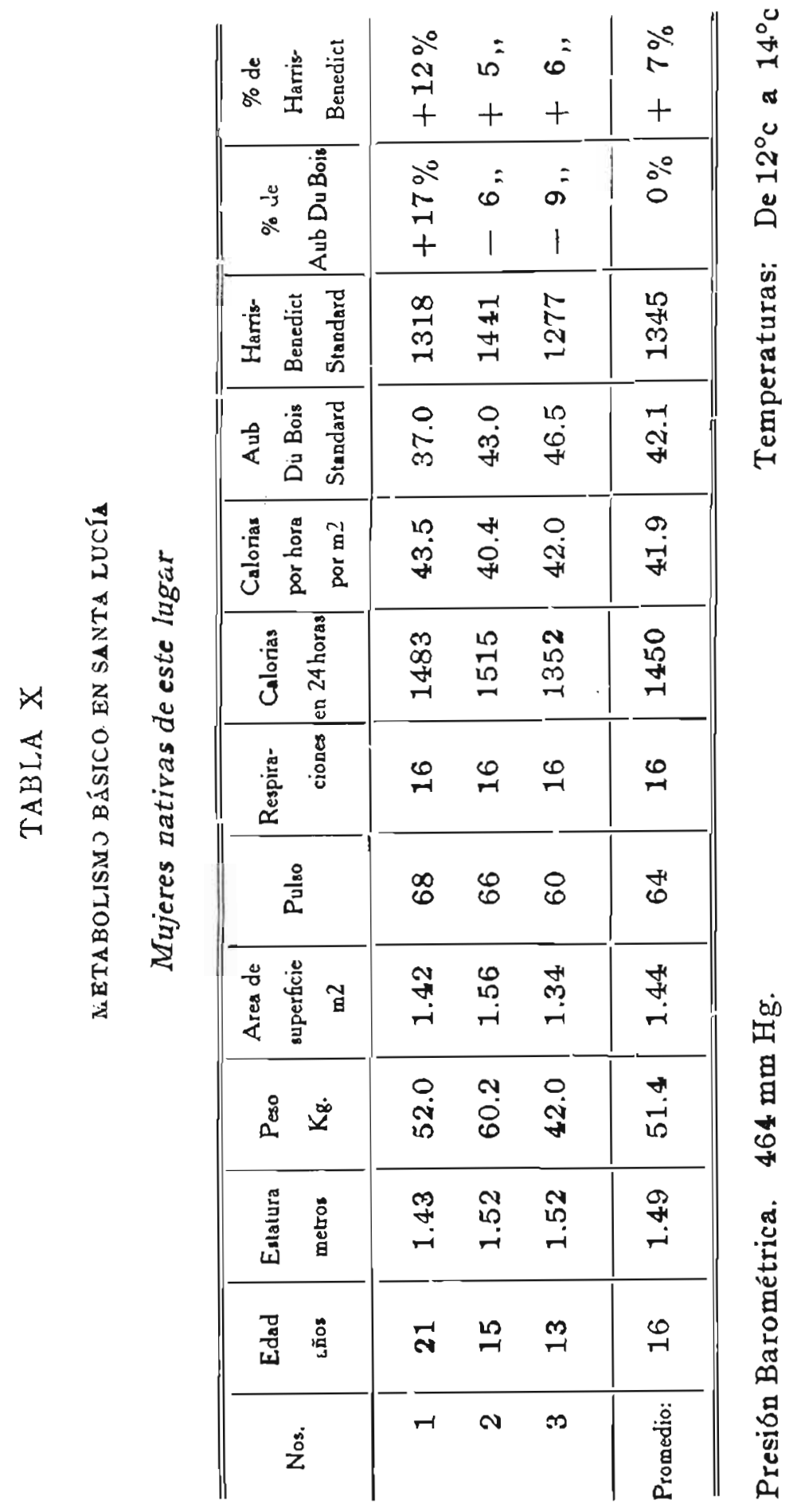


'Por estas determinaciones de mujeres de la edad de I3 hasta 2 I años, vernos que el metabolismo básico se encuentra entre límites normales. Es importante notar que en este grupo de mujeres es donde hay mayor diferencia entre los valotes normales establecidos por AuB-Du Bors y por Harris BeNedict, confirmando así la diferencia de valor atribuido en la producción calórica de las mujeres por estos dos grupos de investigadores.

En las primeras páginas de este trabajo se ha mencionado la acción dinámica específica de las materias alimenticias, o sea el aumento de metabolismo celular por razón de un estimu1o específico que las moléculas alimenticias poseen, siendo este estímulo más marcadio en la proteínas. Pensando que quizá este aumento de metabolismo difería en los nativos de estos altos lugares y en los individuos de raza blanca que viven en planos menos elevados, determinamos esta acción en dos individuos, uno de raza blanca, proveniente de la costa y otro nativo, indígena, de la Oroya. Los dos fueron puestos en completo reposo, determinando el metabolismo básico y en seguida ingerían 100 gramos de carne y un huevo; el metabolismo era calculado media, una y media y dos horas después. En la Tabla XI damos el resultado de esta experiencia. 


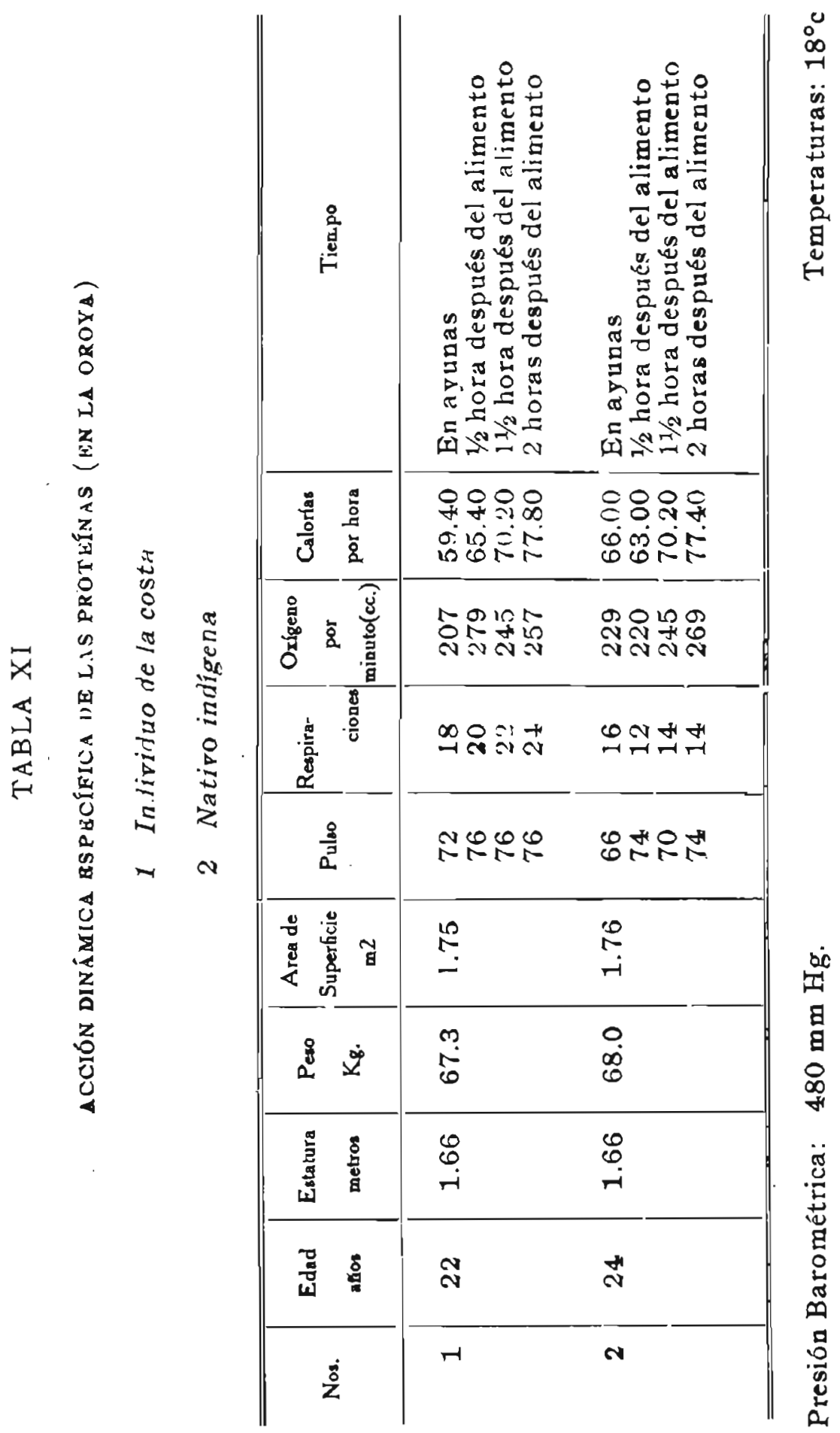


Encontramos que en los dos casos el allmento de metabo lismo seguía líneas paralelas, y por consiguiente no parece haber una diferencia racial en la acción dinámica específica de los alimentos, y tanto el nativo indígena como el individuo de raza blanca respondieron igualmente a la ingestión de la comida. El resultado de ssta experiencia se puede apreciar mejor en el Diagrama 2. 


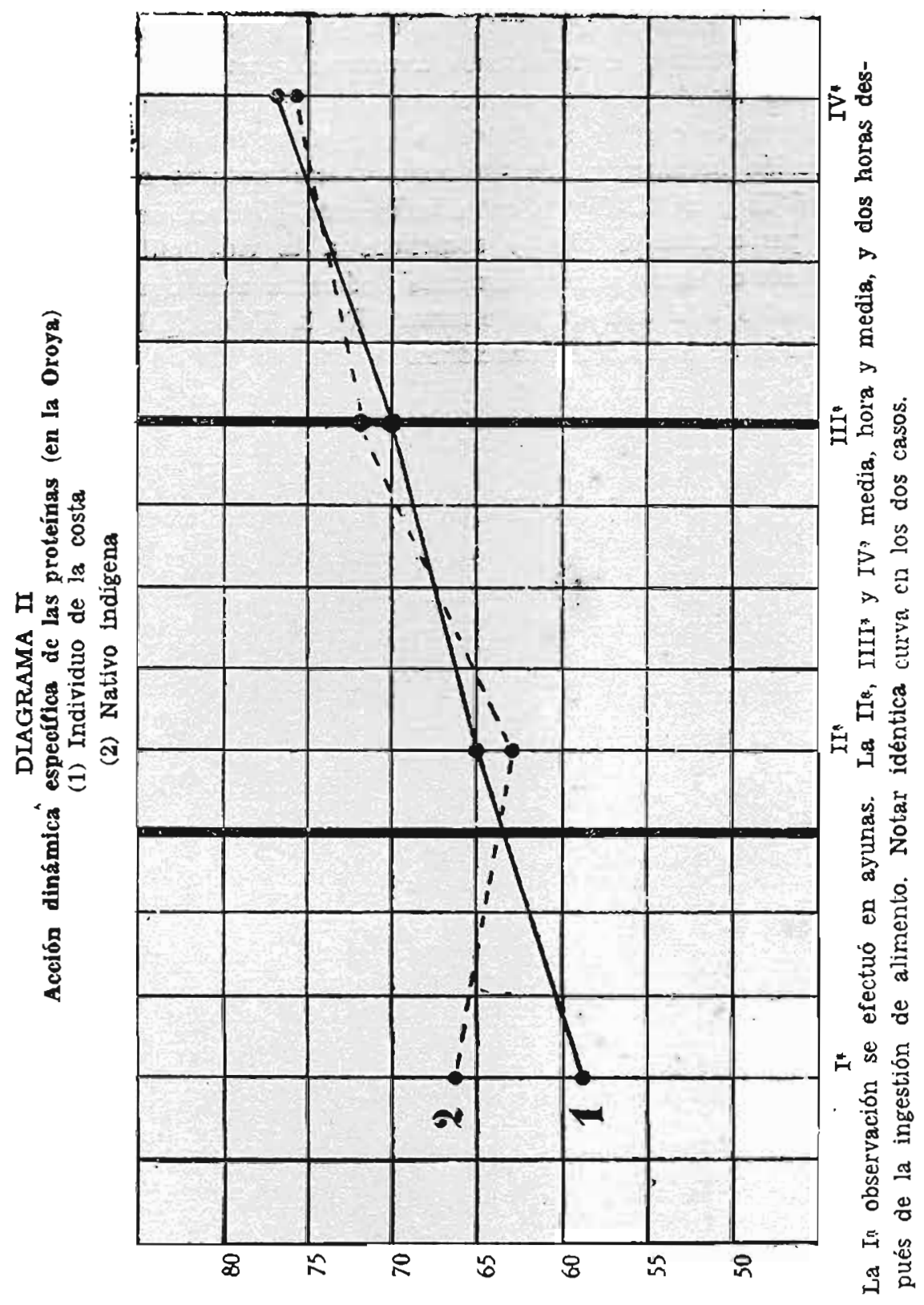

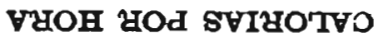


Prosiguiendo los estudios de metabolismo básico en la raza indigena, hemos estudiado un numeroso grupo de soldados nativos de los departamentos del sur y cuya determinación de metabolismo se efectuó en Arequipa a 230 r metros de altura, a una presión barométrica de $576 \mathrm{~mm}$, y temperaturas oscilando entre $16^{\circ} \mathrm{C}$ y $23^{\circ} \mathrm{C}$. Todos estos inclividuos, jóvenes, se encontraban perfectamerite bien y sometidos a la vida militar, de buenos antecedentes $y$ anteriormente dedicados a la agricultura y habituados a la vida en las alturas. En la Tabla XII damos el resultado de estas determinaciones. 


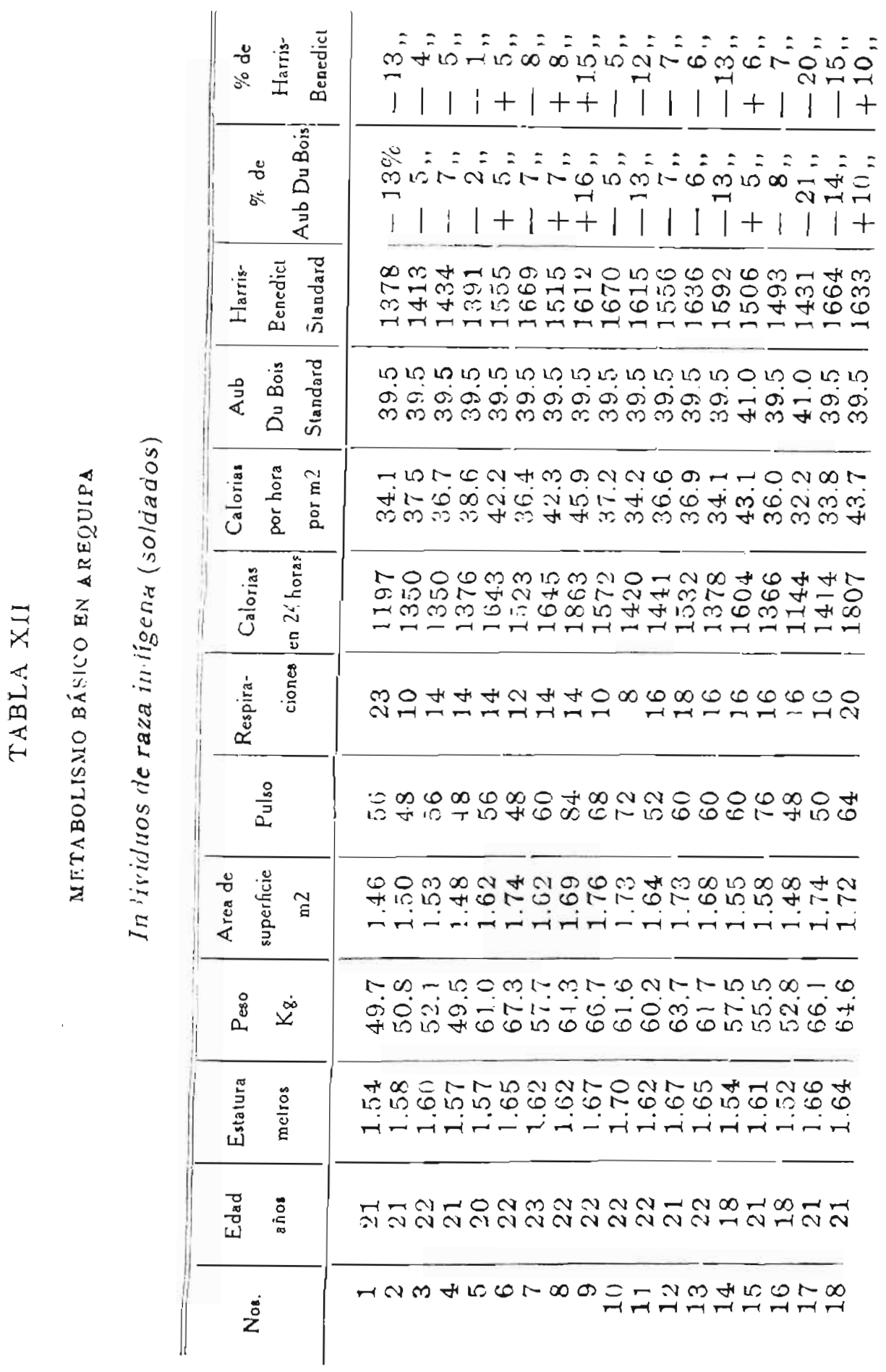




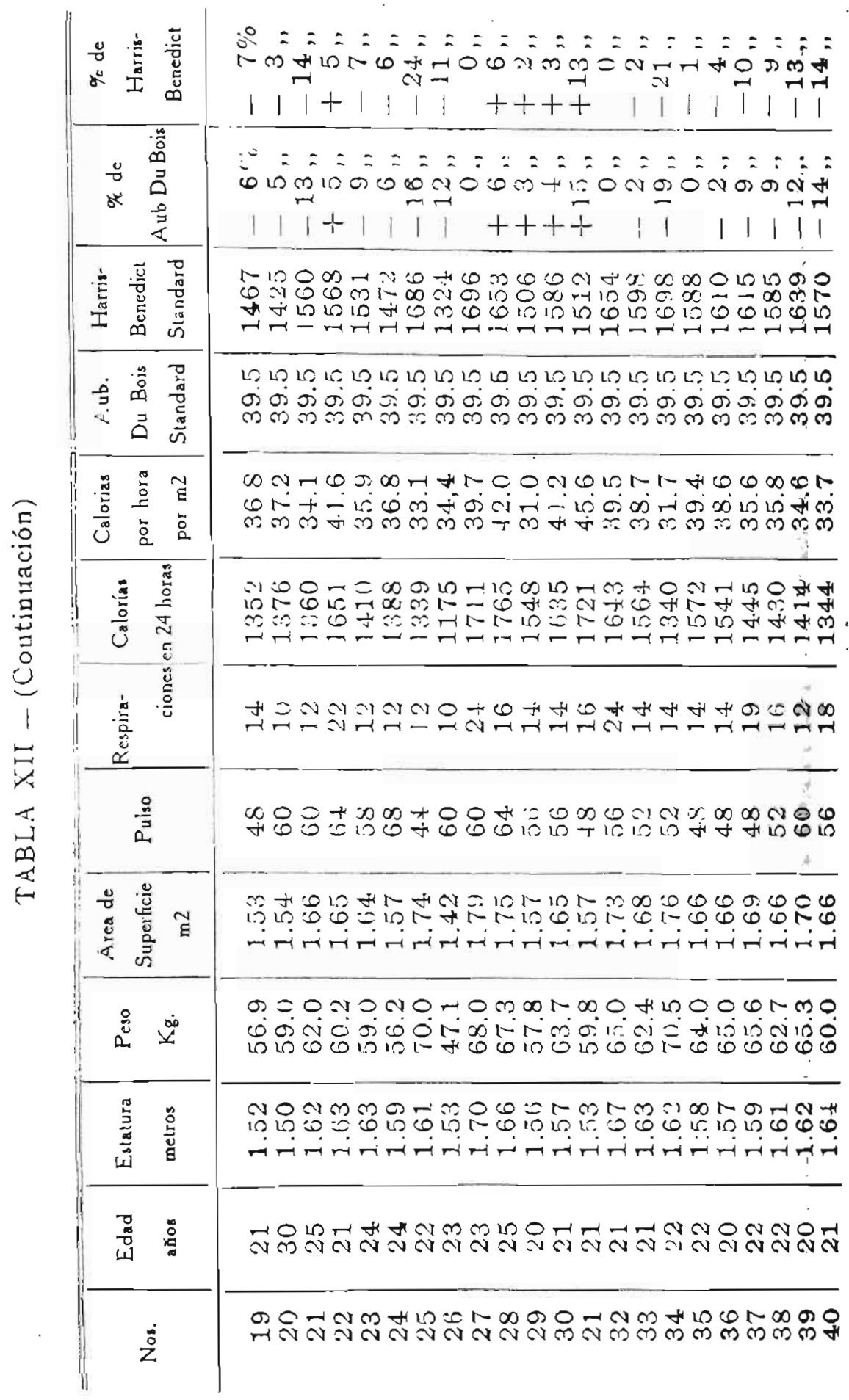


FACULTAD DE MEDICINA

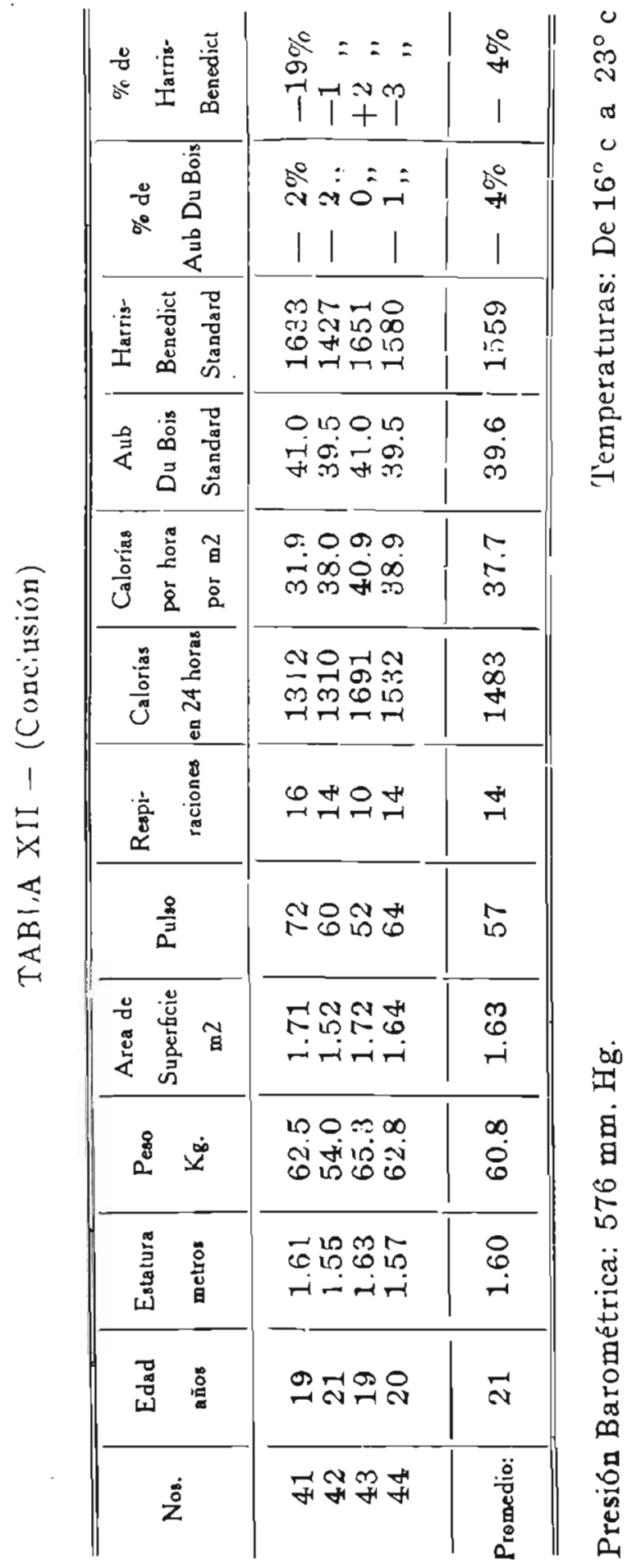


En esta tabla vemos que el promedio general de desviación de los vaiores normales establecidos por Aub-Du Bors y Harris Benedict es de $-4 \%$ en los dos casos, y que el 9 I $\%$ de estos individuos tenía un metabolismo básico entre más y menos I5\%. Sin embargo, es importante notar que algunos de estos individuos, aunque perfectamente normales, poseían un metabolisno bastante disminuído, y por consiguiente es precisc tener en cuenta estú disminución, que podemos llamar fisiológica en algunos individuos que se encuentran en lugares altos. Hay una tenciencia hacia la disminución.

Con este estudio de indígenas en Arequipa, creo que podemos llegar a ciertas conclusiones, que más tarde expresaremos al final de nuestro trabajo. Hemos estudiado el metabolismo de estos indigenas en los diversos lugares que habitan y bajo las diferentes condiciones de vida a que están sometidos.

Quizás es conveniente consignar aquí que siendo el objeto de nuestro estudio el establecimjento de valores normales de iietabolimetría, no hemos incluido nuestras determinaciones de is casos de Eritremia de las alturas, casos señalados por vez primera por el Dr. Carlos Monce, y cuyo metabolismo se encientra en el terrenc de la Patología.

\section{Estudios de Metabolismo Básico en el Trópico}

El estudio del metabolismo básico en el trópico of recia un enorme interés, en vista de las conclusiones de otros investigadores, que mantienen la idea de que los habitantes de estos lugares viven en un nivel metabólico más bajo, Io que les permite adaptarse al desgaste calórico que significa cualquier actividad física en estas regiones de clima tropical, y donde la vida transcurre en una constante laxitud, y con una innata resistencia a cualquier actividad.

En I893, EIJKMAN estudió el metabolismo de hombres blancos y malayos gue viven en los trópicos, y no halló diferencia en el metabolisnio al comparar con los valores establecidos en las clínicas europeas. 
Young, en 1920, estudió el metabolismo de hombres blan$\cos$ que viven a latitud ig sur, y encontró que el metabolismo durante el verano llegaba a un nivel más alto que durante el invierno, y explicó este hecho por la falta de evaporación durante la estación cálicia. En I92I, OzORIO DE AIMEIDA practicando determinaciones en Río de Janeiro (latitud 22.5 Sur) por el método Tissot, en hombres blancos de 23 a 40 años de edad, en una región cuya temperatura media es entre $20^{\circ}$ y $25^{\circ} \mathrm{C}$, con una humedad de 78 a $88 \%$. encontró que por término medio el metabolismo básico es $23 \%$ más bajo que los valores establecidos por AuB-Du Bors, alcanzando los valores más altos en las personas de vida activa. El número de sujetos examinados fué de diez. Iguaimente encontró una disminución en el metabolismo de hombres negros, aunque en menor grado.

En I921, Montoro, estudiando el metabolismo básico de once hombres y cinco mujeres en la Habana, encontró igualmente una disminución en el metabolismo de estos individuos y confirmó el trabajo de Almeida.

KNIPPING, en 1923, estudiando el metabolismo en los trópicos, no halló ninguna alteración, pero un año más tarde, OzORIO DE ALMEIDA, en una nueva investigacióm, encuentra siempre una disminución en el metabolismo de los hombres que viven en la zona tropical de Río de Janeiro, y lo interpreta como un fenómeno de adaptación y como una ventaja en la lucha contra el calor. Igualmente aplicando los valores dados en la fórmula HarRIS-BENEDICT, encuentra una disminución apreciable.

De la revisión de la literatura nos encontramos, pues, ante investigaciones con resultados contradictorios, y era, por tanto, fascinante y atrayente (como lo manifiesta Du Bors) el viaje a las regiones candenteś del norte del Perú, cerca del Ecuador, donde todo el año las poblaciones sufren (he los rigores de un clima cálido. Las determinacionese que siguen han sido tomadas en la ciudad de Piura, que se encuentra a la latitud 5.5 Sur y donde la temperatura durante el invierno oscila entre 20 a $24^{\circ} \mathrm{C}$., y durante el verano de 28 a $34^{\circ} \mathrm{C}$. (en la sombra), con un promedio de humetad bastante alto.

I a observación de los pobladores de estas zonas es bastante interesante: la mavor parte de los nativos acomodan su vida a la menor axtividad física posible, se les ve descansando, ahorrando el menor movimiento, y hasta sus moradas llevan las 
huellas de la miseria que un escaso trabajo les proporciona. En las calles es frecuente ver grupos de estos individuos esperando la ocupación que no llega, y aquellos que trabajan, sean nativos 11 hombres de raza blanca, muestran cierta laxitud y la moclorra del trópico. Era pues, interesante, repetimos, investigar el nivel metabólico de estos individuos, $y$ hallar si a la adaptación voluntaria de inactividad, unen también un nivel metabólico más bajo de actividad celular. Las primeras determinaciones çue damos en la Tabla XIJl fueron tomadas en un grupo de soldados, todos nativos de Piura, cuyo promedio de edad era de 22 años. 


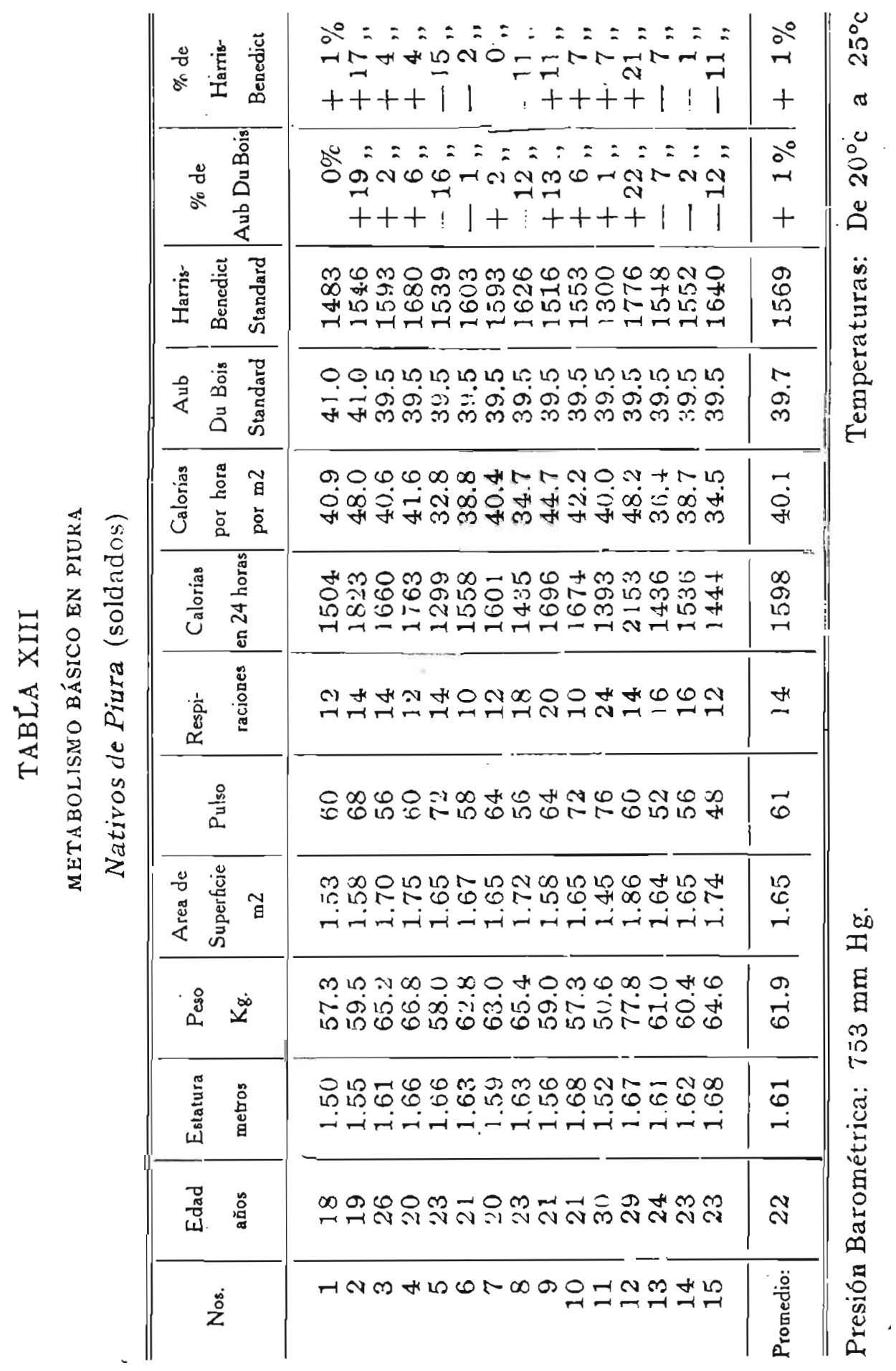


Por esta tabla vemos que el término medio de las variaciones en las distintas determinaciones, aplicando las fórmulas de Aub-Du Bors y Harris Benedict, es solamente de $+\mathrm{I} \%$ y por consiguiente se encuentra en un límite completamente normai, y no hallamos la disminución en el metabolismo, como lo afirman otros investigadores. Sin embargo, pensando que quizás estos individuos, como pertenecientes al ejército y llevando una vida activa, pueden modificar su nivel metabólico por la dicha actividad, escogimos otros individuos, todos nativos d'e estos lugares, de vida sendentaria y de poca actividad, cuya edad por término medio era de 20 años, pudiendo así compararse con el otro grupo. En la Tabla XIV damos los resultados de estas determinaciones. 


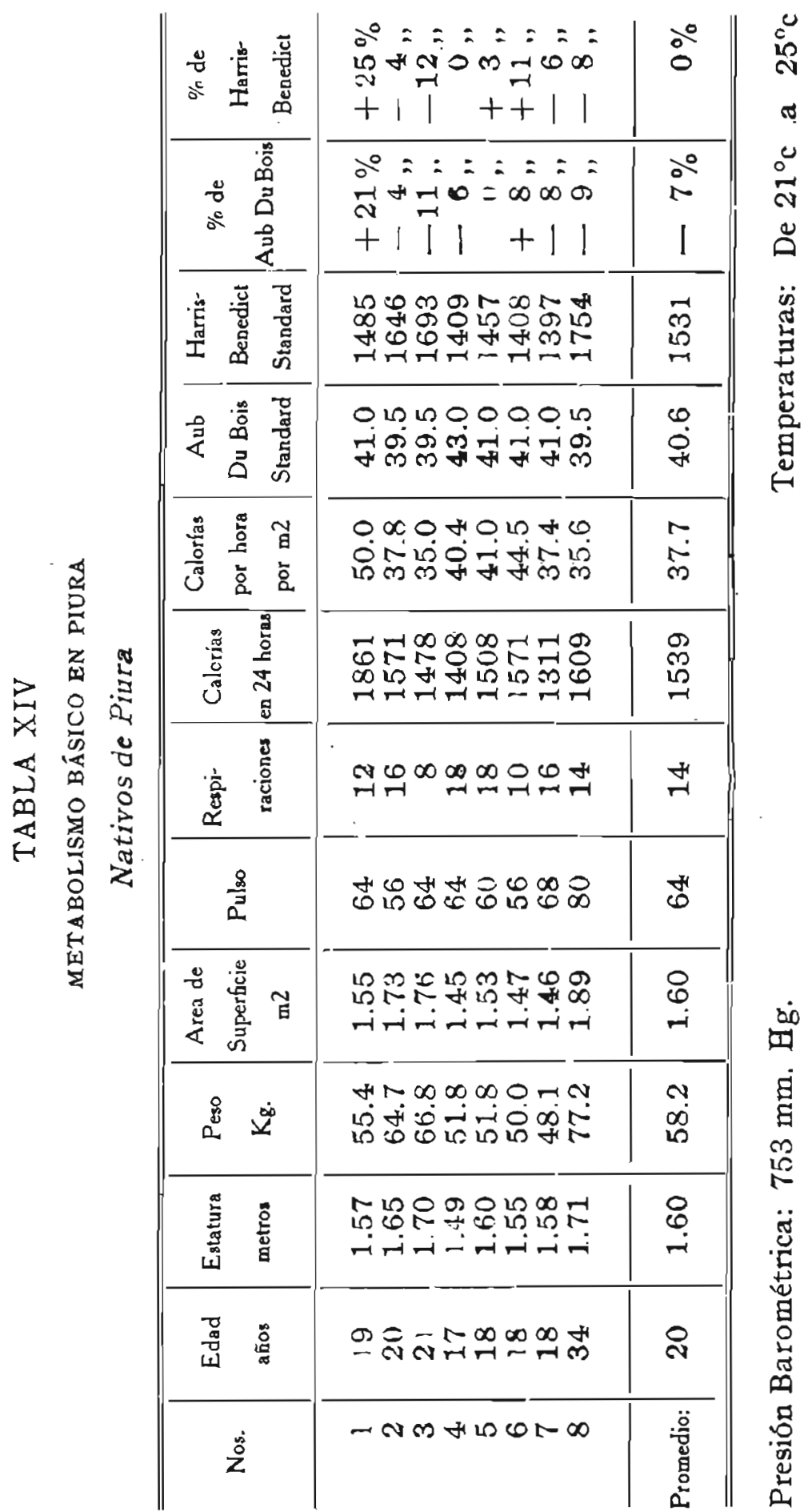


Los resultados de estas determinaciones igualmente confirman las obtenidas con el otro grupo de nativos pertenecientes al ejército, es decir que el metabolismo básico presenta variaciones entre los límites normales, y no encontramos la disminución pretendida por previos investigadores, pudiendo concluír en que estos individuos habitantes de los trópicos viven en el mismo nivel metabólico de otros centros y de otros climas, y que en su lucha contra el calor tratan de adaptarse, reduciendo su actividad física hasta un rínimum, pero sin que exista una disminución de actividad orgánica celular.

En el capítulo de estudio sobre el metabolismo básico en las alturas, se encuentra una experiencia sobre el desgaste calórico a determinado ejercicio físico, y recordamos que tanto el nativo de esos !ugares. como el individuo de la costa acusaban un aumento de metabolismo (más marcado en el primero) seguido de una clisminución que podría ser interpretada como una economía compensadora. Aquí en Piura repetimos la experiencia con un nativo de este lugar tropical y como se ve en la Tabla $X V$, en que damos los resultados, hay un aumento apreciable del metabolismo innediatamente después, aumento que persiste tras un período de quince minutos, y no encontramos la disminución hallada en la Oroya con igual experiencia. 


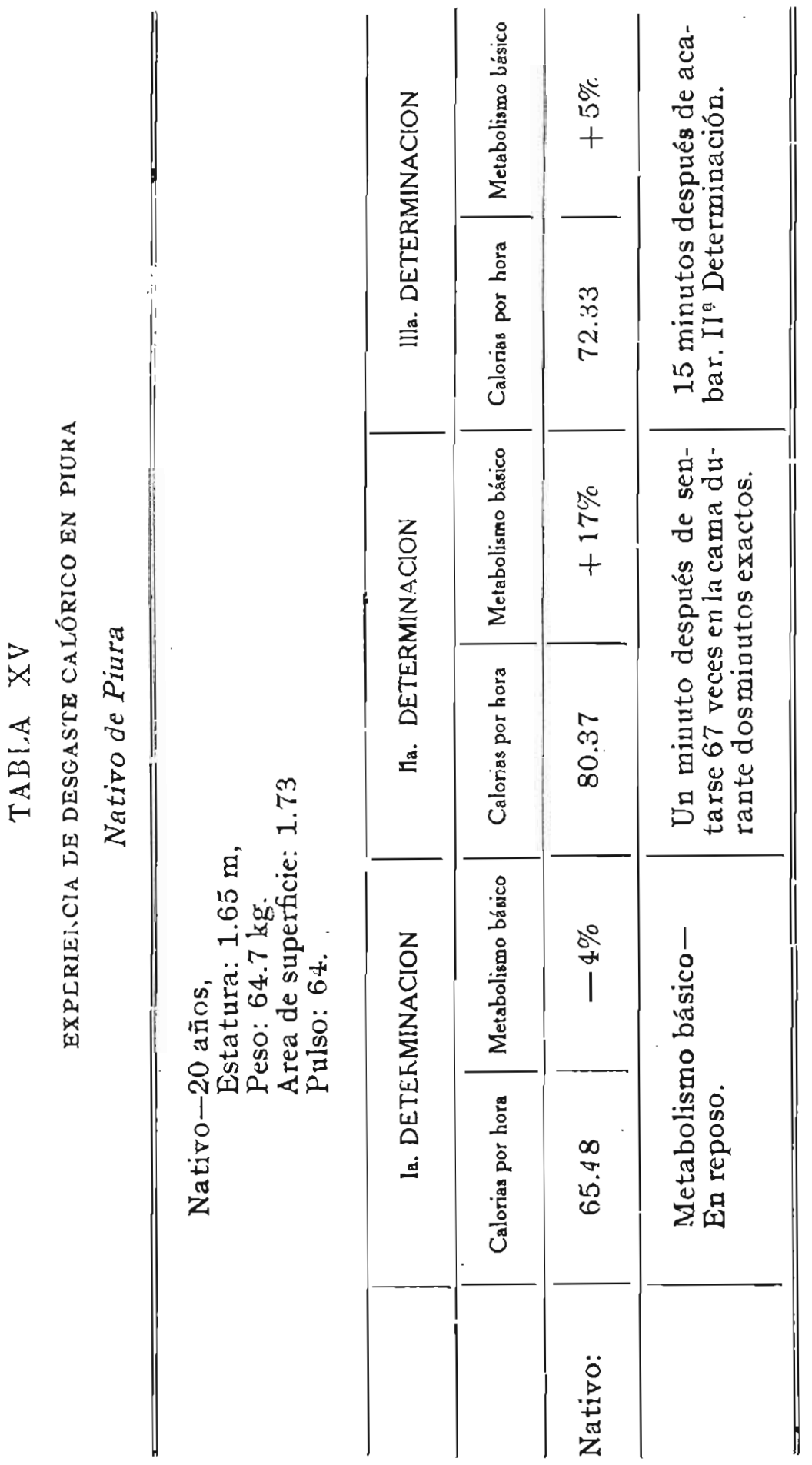




\section{Resumen y Conclusiones}

I-En el presente trabajo se ha determinado el metabolismo básico en divcrsas regiones del Perú, en poblaciones al nivel del mar, en diferentes alturas y en las regiones tropicales.

Se ha practicado 167 determinaciones (no tomando en cuenta muchas elimi!larlas por cooperación imperfecta), usando el método de calorimstría inciirecta, o sea el consumo de oxigena en los aparatos de SANBorn y Benenict Roth con el método gráfico.

2-La raza blanca de nuestras poblaciones posee el mismo nivel metabólico de otras razas, y por consiguiente son aplicables los valores normales establecidos en otros centros.

3-La raza indigena, a pesar de sus caracteres raciales y -ntronométricos completainente clistintos a utras razas, y a pesar de su perfecta adaptación a la vida en las alturas y su enorne resistencia física y hábitos de vicla completamente diferentes, igualmente posee el mismo nivel metabólico de otras razas y 1.s determinaciones de metabolismo básico caen entre los límites normales de variación.

Esta conclusión se aplica a inclividuos tanto en poblaciones al nivel del mar, como en las diferentes alturas y en el trópico.

4-Ios individuos que sufren de "soroshe" o mal de las alturas, al ascender a lugares altos, prescntan una reducción considerable del metabolisno, disminución que st; aparta completamente de los límites normales de variación.

Esta disminución se encuentra igualmente presente, aunque en menor grado. en los individuos de residencia más prolongrada en estos altos lugares, pero que padecen de ciertos sintomas de inadaptación.

5-Esta reducción de metabolismo en los individuos que sufren de sintomas cle inadaptación en las alturas parece ser un fenómeno compensatorio. de manera que cuando el organismo sufra el enorme rlesuste calórico que cualquier actividad física carsa, en estos altos lugares. cuncuentre al organismo a un nivel metahólico más apropiado para sufrir esta pérdicla de energía.

6-Tos nations do ectns altos lugares que no experimentan síntoma alguno con cualcunier actividlad física por violenta que esta sea, presentan un menor desgaste calórico, comparados com 
el individuo de la costa que se fatiga, al someterlos a idéntico ejercicio físico, y ambos presentan después una reducción en el metabolismo.

Este menor gasto de energía en la raza indígena explica quizás en parte su perfecta adaptación a la vida en las alturas.

7 La acción dinámica específica de las proteínas en los individuos de raza indígena y en el nativo de la costa, presenta iguales caracteres.

8-El número de pulsaaiones por minuto se encuentra aumentado en las alturas.

9-El metabolismo básico en los individuos habitantes del trópico se encuentra entre límites completamente normales, y no hallamos la disminución que afirman otros investigadores.

Esta conclusión se aplica tanto a los individuos de vida activa como a aquellos de vida sedentarià.

Io-Los valores normales establecidos por Aub-Du BoIs y los dados por Harris-Benedict son igualmente aplicables en nuestro medio, y las variaciones entre ellos son insignificantes.

I I-Siendo más fácil y rápida la comparación con los valores normales clados por la escalla de AuB-Du Bors, recomendamos su adaptación entre nosotros, y que sirva de base para todos los futuros estudios y determinaciones de Metabolimetría en nuestro medio, cualquiera que sea la raza y el sexo del individuo metabolizado, y cualquiera que sea el lugar donde se verifique la determinación, con solamente la excepción del individuo no adaptado a la vida en las alturas. en el que la disminución del metabolismo parece ser un fenómeno fisiotógico compensador.

12-Entre nosotros las variaciones normales de los valores estableciclos en la escala de $\mathrm{A} \mho-\mathrm{Du}$ BoIs se encuentran entre más $\mathrm{y}$ menos $15 \%$ y por consiguiente estos son y serán los límites de la normalidad en nuestras determinaciones. 


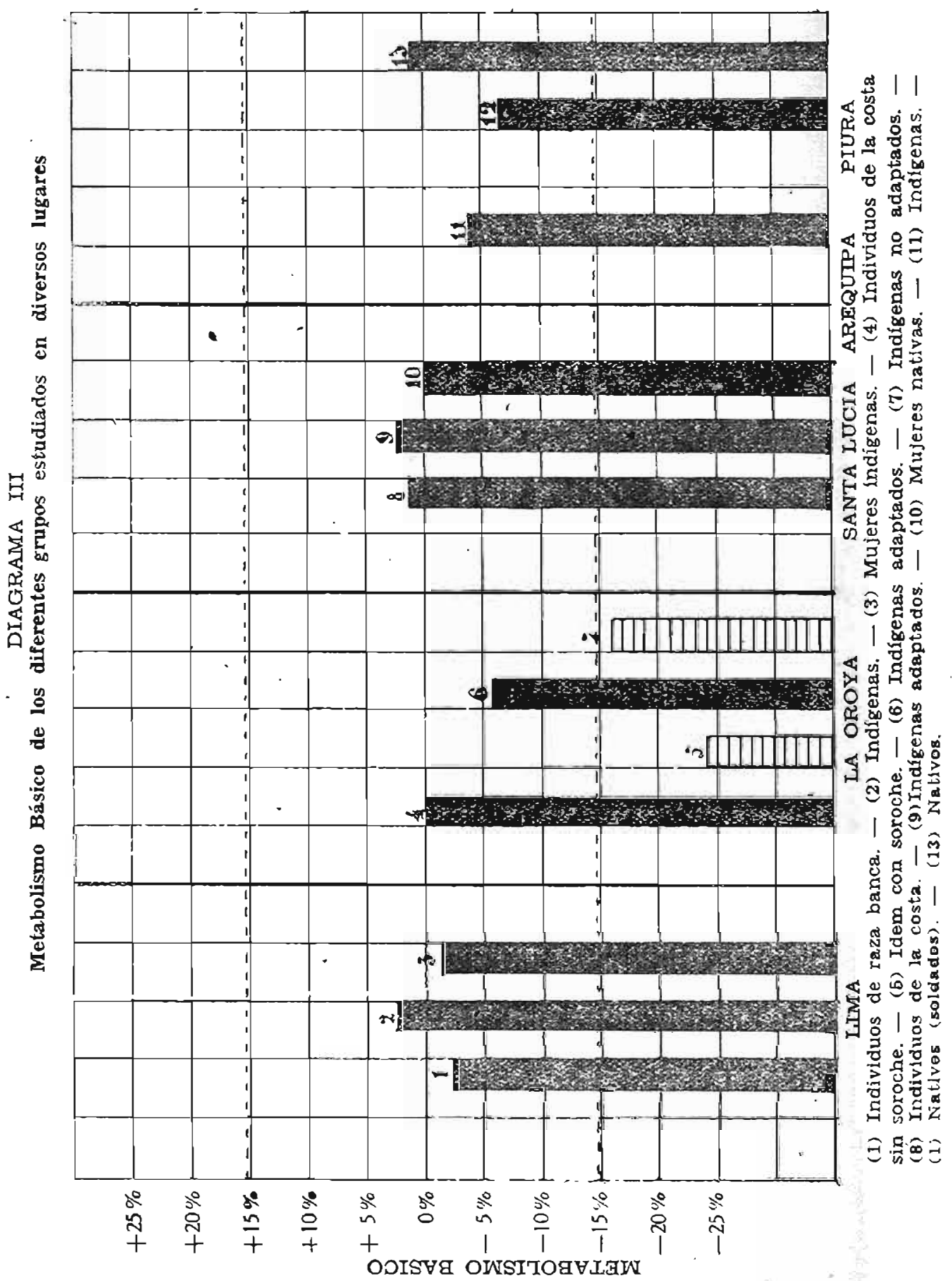




\section{BIBLIOGRAFIA}

Regnault Rieset.-An. de chime et phys.--París 1849 , 26, 299;

Petten kofrer, Voit.-Ztschr. f. biol. i867, 3, 380;

Maginus Levy.-Berl, klinh. Wchnschr. I895, 32, 650;

Eijkman.-Virchw's Arch. f. path. Anat. I893, CXXXIII, 100 ;

Von Schrotter, Zuntz.-Pfluger's archiv. I902. XCII, 479;

DURIG, Zunxz.-Archiv. fur Phys. I904, 4r7;

Lindifard.-Skan. archiv. f. Physiol. I9I2, 26, 221;

Douglas, Haldane, Henderson, Scheneider.-Phil Tr. I913, Ser. B CCIII, I86;

Hill.-Gov. Reprts. I9I4, No. I00;

Du Bors, Du Bors.-Arch. Int. Med. I915, XV, 868;

Benedict.-J. Biol, Chem. I9I5, XX, 263;

Hasselback, LindHard.—Biochem. Ztschr. I9I 5 , LXVIII, 265 ;

Benedict, Smith.-J. Biol. Chem. I9I5, 20, 243;

Benedict, Roth.-J. Biol. Chem. I915, 20, 23I ;

Benedict, Emmes.-J. Biol. Chem. I9I 5, 20, 253;

Benedict.-J. Biol. Chem, I9I 5, 20, 263;

Du Bors, Du Bors.—Arch. Int. Med.-rg16, XVII, 863;

Beredict.-Am. J. Ohysiol. rgi6, XLI, 275;

Aub-Du Bors.-Arih. Int. Med. 19r7, VIX, 823;

Lusk- The Science of Nutrition. I917.

Harris, Benedict, -Carnegie Inst. Nash. r9I9. Publ. 279;

Dreter.-Lancet, I920, II, 289;

Ozorio de Almeida.-J. de Physiol, et de path, gen. Igzo, XVIII, †I2;

Ozorio de Almeida.-Item 1920, XVIII, 958;

Young.-Ann. Trop. Med. and Paras. I920, XIII, 3I3;

Lusk.-Physiol, Rev. I92I, I, 523; 
Faber, Melcher.-Ptoc. Soc. Exp. Biol. and Med.-I92I, $\mathrm{XIX}, 62$;

Iontoro.-V Congreso de Medicina. Habana I92I.

Boothby, Sandiford.-J. Biol. Chem. 1922. LIV, 767;

Hill, Ca M PBell.—Breit. Med. J. 1922, I, $30 \mathrm{I}$;

OKada, Sakural y otros.-Ikahi Jiho. No. I479, I922;

Barcroft, Binger, Bock y otros.--Phils. Tr. Royal Soc. London 'Ser. B, Vol. 2I I ;

Peterson, Walter.--J. A. M. A. 78,3411, I922;

Schneider.-A. M. J. Physiol. I923, LiXV, I07;

FLem MinG.-J. Met Research, 4,I95, 1923;

Krogh.-Boston Med and Surg. J. I89, 3133, 1923;

Ozorio de Almeida.-J. de Physiol, et de path. gen. Tome XXII, I2, 1924;

Mc Cann.-Medicine, Vol. IIII, I, I924é

Du Bors.-Basal Betabolism in Health and Disease. 1924.

TAKAHIRA.-Report of the Imperial Nutrition. Institute of Japjan, II, I925;

Mc Cleod, Crofts, Benedict.-Am. J. Physiol, 79: 221, I926;

Hafkesbring, Borgstrom.-Am. J. Physiol, 79: 221 ; 1926;

IURner.- J. A. M. A. 87, 2052, I926;

OKada, Sakurai, Kameda.-Arc. Int. Med. 38,590, 1927. 